\title{
Structural Analysis of Monoclonal Antibodies with Top-down and Middle- down Electron Transfer Dissociation Mass Spectrometry: The First Decade
}

\author{
Luca Fornellia, Daniel Ayoubb, Kristina Srzentićc ${ }^{\mathrm{c}}$, Konstantin O. Nagornov ${ }^{\mathrm{d}}$, Anton N. Kozhinov ${ }^{\mathrm{d}}$, \\ Natalia Gasilova ${ }^{\mathrm{e}}$, Laure Menin ${ }^{\mathrm{e}}$, Alain Beck ${ }^{\dagger}$, and Yury O. Tsybin ${ }^{\star d}$
}

\begin{abstract}
Monoclonal antibodies (mAbs) are protein biotherapeutics with a proven efficacy toward fighting lifethreatening diseases. Their exceptional healing potential drives the annual increase in the number of novel mAbs and other antibody-like molecules entering clinical trials and the number of approved mAb-based drugs. Mass spectrometry (MS) offers high selectivity and specificity for the potentially unambiguous identification and comprehensive structural characterization of proteins, including at the proteoform level. It is thus not surprising that MS-based approaches are playing a central role in the biopharma laboratories, complementing and advancing traditional biotherapeutics characterization workflows. A combination of MS approaches is required to comprehensively characterize mAbs' structures: the commonly employed bottom-up MS approaches are efficiently complemented with mass measurements at the intact and subunit (middle-up) levels, together with product ion analysis following gas-phase fragmentation of precursor ions performed at the intact (top-down) and subunit (middle-down) levels. Here we overview our group's contribution to increasing the efficiency of these approaches and the development of the novel strategies over the past decade. Our particular focus has been on the topdown and middle-down MS methods that utilize electron transfer dissociation (ETD) for gas-phase protein ion fragmentation. Several approaches pioneered by our group, particularly an ETD-based middle-down approach, constitute a part of commercial software solutions for the mAb's characterization workflows.
\end{abstract}

Keywords: Antibody-drug conjugate · Drug-to-antibody ratio · Electron transfer dissociation · Fourier transform mass spectrometry $\cdot$ Orbitrap $\cdot$ Proteoform
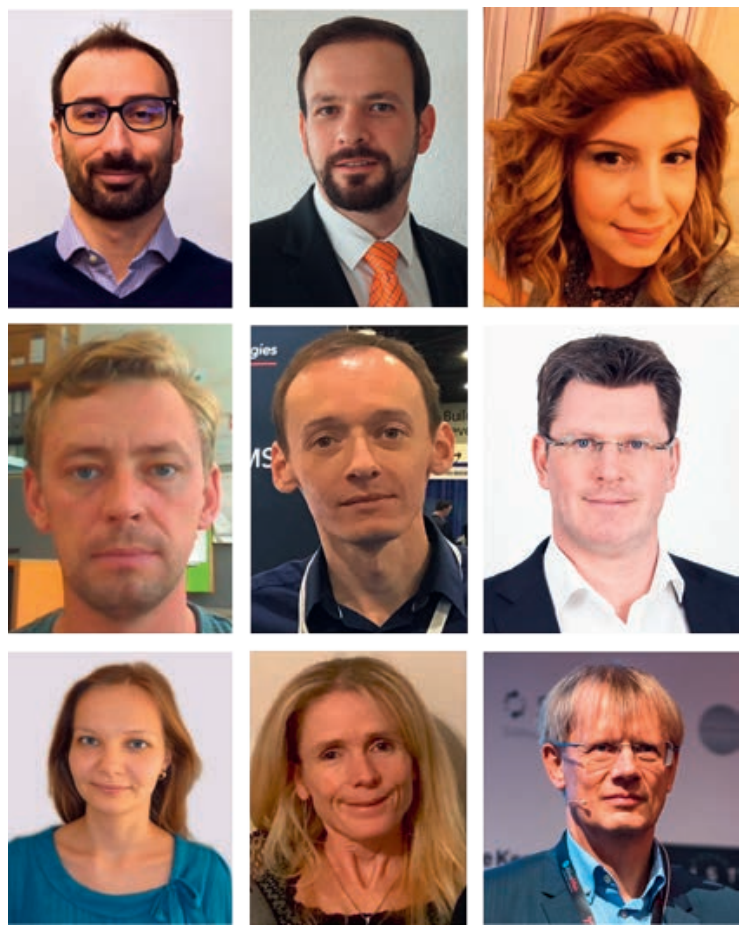

This mosaic portrays the authors of the manuscript. All are involved in mass spectrometry method and technique development in general, and top-down mass spectrometry-based monoclonal antibodies structural analysis in particular. From left to right: (the first row) Luca Fornelli, Daniel Ayoub, Kristina Srzentic, and (the second row) Konstantin Nagornov, Anton Kozhinov, and Yury Tsybin (assistant professor, laboratory head) were all members of the Biomolecular Mass Spectrometry Laboratory (LSMB) at Ecole Polytechnique Fédérale de Lausanne (EPFL), until 2014. Since 2014 their top-down mass spectrometry research program has been undertaken and advanced at the LSMB spin-out company, Spectroswiss. Authors continued, from left to right, the third row: Natalia Gasilova, Laure Menin, and Alain Beck have been external collaborators for the monoclonal antibody research program first at LSMB and currently at Spectroswiss.

\section{Introduction}

Monoclonal antibodies (mAbs) are large proteins produced by a single B-cell clone that demonstrate exceptional abilities in the fight against a variety of pathologies. ${ }^{[1]}$ Unlike small molecule therapeutics, mAbs and their derivatives are complex bioproducts that are very heterogeneous and are present in many proteoforms, particularly glycoforms. By definition, proteoforms encompass the chemical diversity of proteins, that arises due to the combinations of genetic polymorphism, ribonucleic acid (RNA) splice variants, and

${ }^{*}$ Correspondence: Dr. Y. O. Tsybind, E-mail: tsybin@spectroswiss.ch aDepartment of Biology, The University of Oklahoma, Norman, OK 73019, USA; 'اchnos Sciences S.A., CH-2300 La Chaux-de-Fonds, Switzerland; ' Thermo Fisher Scientific, CH-4153 Reinach, Switzerland; d'Spectroswiss, EPFL Innovation Park, Building I, CH-1015 Lausanne, Switzerland; eEcole Polytechnique Fédérale de Lausanne, CH1015 Lausanne, Switzerland; 'Centre d'Immunologie Pierre Fabre, 74160 St Julien-en-Genevois, France 
post-translational modifications (PTMs). ${ }^{[2]}$ In the biotherapeutic drug development process, many of the mAbs' proteoforms need to be identified and characterized to comply with the regulatory authorities' guidelines, including safety requirements.

The biotherapeutics-grade mAbs are generally from the immunoglobulin $\mathrm{G}(\mathrm{IgG})$ class or isotype. ${ }^{[3]}$ Structurally, an individual $\mathrm{IgG}$ molecule is a tetrameric glycoprotein complex composed of two $\sim 50 \mathrm{kDa}$ heavy chains (Hc) and two $\sim 25 \mathrm{kDa}$ light chains (Lc) (Fig. 1). Four IgG subclasses exist and are defined by their $\mathrm{Hc}$ amino acid sequence: IgG1, IgG2, IgG3, and IgG4. The IgG3 mAbs are not (yet) used as therapeutics due to their significantly faster biological clearance rates than that of the IgG1/IgG2 subclasses. ${ }^{[4]}$ The threedimensional structures of $\mathrm{mAbs}$ are maintained by disulfide bridges (16 for IgG1 and IgG4; 18 for IgG2) and non-covalent interactions. The Lc and Hc subunits are linked by a single disulfide bond and the two Hc subunits by two (for IgG1 and IgG4) or four (for IgG2) disulfide bonds located in a short hinge domain. ${ }^{[5]}$ The other 12 cysteine bridges are intramolecular and define six different globular domains, both variable and constant (Fig. 1). MAbs fulfill their role by binding foreign substances (antigens) using the variable domains represented predominantly by the three loops connecting individual $\beta$-strands in each of the complementarity determining regions (CDRs). Like natural IgGs, all recombinant mAbs contain an -Asn-X-Ser/Thr-Yconsensus sequence for $\mathrm{N}$-glycosylation in their $\mathrm{Hc}$ constant domain (where $\mathrm{X}$ and $\mathrm{Y}$ are amino acids different from proline). As a result, a typical mAb-based biotherapeutic is represented by a defined set of proteoforms (glycoforms).

Despite the powerful therapeutic potential of the mAbs in the most common monospecific bivalent configuration (two identical Lc and two identical $\mathrm{Hc}$ ), current $\mathrm{mAb}$ development strategies consider increasing the mAb-derived biotherapeutics potency by diversifying

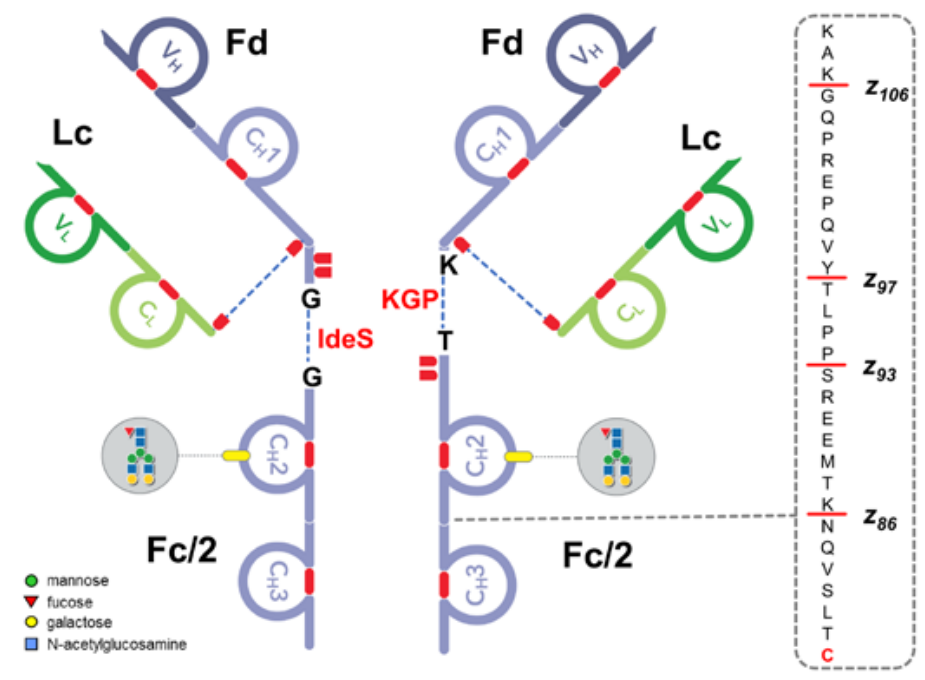

Fig. 1. The mAb puzzle: structural organization of IgG1. A pair of light chains (Lc, shown in green) is connected with a pair of heavy chains (Hc, shown in blue) via disulfide bonds (shown with red ovals). Each Lc contains a variable, $V_{1}$, and a constant, $C_{1}$, structural domain. Each $\mathrm{Hc}$ contains a single variable, $\mathrm{V}_{\mathrm{H}}$, and three constant, $\mathrm{C}_{\mathrm{H}} 1-3$, structural domains. The main proteoforms are due to the $\mathrm{N}$-glycosylation of Asn in the Hc constant domain. The two heavy chains are shown split into halves by structure specific enzymes: IdeS (cleaves mAbs below the hinge region) and KGP (cleaves $\mathrm{mAbs}$ above the hinge region). The corresponding $\mathrm{Hc}$-derived subunits are $\mathrm{Fd}$ (the $\mathrm{N}$-terminal part of $\mathrm{Hc}$ ) and $\mathrm{Fc} / 2$ (the C-terminal part of $\mathrm{Hc}$ ). The inset at the right shows a part of $\mathrm{Hc}$ sequence with the locations of preferential backbone cleavage sites upon ETD of a human anti-rhesus D mAb analysis. ${ }^{[29]}$ the roles that their variable domains play, and by conjugation of mAbs with different drug molecules. ${ }^{[6]}$ The former is realized via the development of multi-specific mAbs (e.g., multi-specific mAbs that carry different binders), ${ }^{[7]}$ and the latter has given rise to a new class of biotherapeutics, the antibody-drug conjugates (ADCs). ${ }^{[8]}$ The currently employed drugs (payloads) range from small, less than $1 \mathrm{kDa}$, molecules to large, several kDa, drugs, such as oligonucleotides. The ADCs carrying oligonucleotides can also be referred to as antibodyoligonucleotide conjugates (AOC). ${ }^{[8 e]}$ Structural analysis of ADC/ AOC samples includes quantitative analysis of the conjugated drug profile heterogeneity - a distribution showing how many drugs are attached to a given $\mathrm{mAb}$ and the average value of this distribution. This information is known as the drug-to-antibody ratio (DAR), with the average DAR values for modern ADCs varying from 2-10 and even beyond that ${ }^{\left[{ }^{9]}\right.}$ DAR monitoring constitutes a critical quality attribute (CQA) in ADC analysis (Fig. 2). ${ }^{[10]}$

Mass spectrometry (MS) is a powerful analytical technique enabling comprehensive mAb structural characterization. ${ }^{[8 c, 11]}$ Nowadays, MS techniques are implemented throughout all stages of the mAb-based biotherapeutics' life cycle - from discovery to development, production, validation, and storage. ${ }^{[3,8 c, 12]}$ Due to its high specificity and selectivity, as well as its ability to monitor several molecular quantities simultaneously and directly, MS is considered as a complementing technology to the proven biotherapeutics release and characterization methods, such as capillary electrophoresis and liquid chromatography (LC). [10b,13]

Based on the structural details of mAbs and mAb-derived biotherapeutics (Fig. 1), the following information needs to be provided by the MS-based approaches. First, an accurate determination of the mass of the mAb (or its subunits) to determine, based on the deposited amino acid sequence, potential global variations such as presence of chemical modifications, followed by validation of the amino acid sequence. This analysis shows the structural completeness (integrity) of a mAb molecule in the development or production process. Second, a relative percentage of the main glycoforms (proteoforms) needs to be deduced (glycosylation profiling). Third, expected and unexpected modifications (including deamidation, isomerization, oxidation, and glycation) should be identified, quantified and, preferably, localized. Fourth, the integrity and scrambling of the inter- and intra-molecular disulfide bonds needs to be controlled. ${ }^{[14]}$ Further analyses may include charge variant analysis, de novo sequencing, light and heavy chain pairing, estimation of the DAR values for ADC/AOC samples, determination of the extent of $\mathrm{mAb}$ aggregation, and conformation analysis. ${ }^{[8 \mathrm{~b}, 15]}$

The MS approaches for mAb/ADC analysis have greatly evolved over the past decade. ${ }^{[3,12 b, 16]}$ Major benefits have resulted from developing high resolution and high mass accuracy MS approaches in combination with the enhanced overall capabilities of modern mass spectrometers in their ability to ionize, transfer, and detect ions of high mass and $m / z$ values.[17] The development of native MS stands out as a particularly enabling technology. ${ }^{[18]}$ An equally important driver for the success of MS-based workflows is developing tandem mass spectrometry (MS/MS) methods for gas-phase protein ion activation and dissociation. ${ }^{[19]}$ The originally employed ergodic methods, e.g., resonant and beam-type collision-induced dissociation (CID), have been complemented with the non-ergodic methods, such as electron capture and transfer dissociation (ECD/ETD). ${ }^{[20]}$ In addition, a whole arsenal of other ion activation and dissociation techniques have been developed or tailored for the analysis of mAbs, including in-source decay in matrix assisted laser-desorption ionization (MALDI ISD), [21] ultraviolet photodissociation (UVPD), ${ }^{[22]}$ and surface-induced dissociation (SID). ${ }^{[23]}$ Other gas-phase ion chemistry processes, such as proton-transfer reaction (PTR) or proton-transfer charge reduction (PTCR), ${ }^{[24]}$ have shown their benefits in manipulating the highly complex product ion populations generated by gas-phase fragmentation of these large proteins. Finally, parallel discoveries 


\begin{tabular}{|c|c|}
\hline \multirow{4}{*}{$>150 \mathrm{kDa}$} & Species: intact ADCs and AOCs \\
\hline & $\begin{array}{l}\text { Sample preparation: none, desalting, } \\
\text { deglycosylation }\end{array}$ \\
\hline & $\begin{array}{l}\text { CQAs: major glycosylations, DARs, PTMs, } \\
\text { integrity }\end{array}$ \\
\hline & $\begin{array}{l}\text { Methods: intact mass measurements by } \\
\text { native and denaturing MS via direct infusion, } \\
\text { RPLC-MS, SEC-MS }\end{array}$ \\
\hline \multirow[t]{3}{*}{$\sim 150 \mathrm{kDa}$} & Species: intact antibodies \\
\hline & $\begin{array}{l}\text { deglycosylation } \\
\text { CQAs: major glycosylations, limited } \\
\text { sequencing, integrity }\end{array}$ \\
\hline & $\begin{array}{l}\text { Methods: intact mass measurements by } \\
\text { native and denaturing MS via direct infusion, } \\
\text { RPLC-MS, SEC-MS; top-down analysis with } \\
\text { HCD/ETD/UVPD, ... }\end{array}$ \\
\hline$\sim 100 \mathrm{kDa}$ & $\begin{array}{l}\text { Species: mAb/lgG subunits }\left(\mathrm{F}(\mathrm{ab})_{2}\right) \\
\text { Sample preparation: structure-specific } \\
\text { digestion (e.g., IdeS) } \\
\text { CQAs: limited sequencing, major } \\
\text { glycosylations, PTMs } \\
\text { Methods: middle-up mass measurements } \\
\text { by direct infusion, RPLC-MS, SEC-MS; } \\
\text { middle-down analysis with HCD/ETD/UVPD }\end{array}$ \\
\hline \multirow{4}{*}{$\sim 50 \mathrm{kDa}$} & Species: $\mathrm{mAb} / \mathrm{lgG}$ subunits $(\mathrm{F}(\mathrm{ab}), \mathrm{Fc}, \mathrm{Hc})$ \\
\hline & $\begin{array}{l}\text { Sample preparation: S-S bond reduction, } \\
\text { structure-specific digestion (KGP, IdeS, } \\
\text { papain, etc.) }\end{array}$ \\
\hline & $\begin{array}{l}\text { CQAs: chain pairing, limited sequencing, } \\
\text { large PTMs }\end{array}$ \\
\hline & $\begin{array}{l}\text { Methods: middle-up mass measurements: } \\
\text { direct infusion and RPLC-MS; middle-down } \\
\text { analysis with HCD/ETD/UVPD, .. }\end{array}$ \\
\hline \multirow[t]{4}{*}{$\sim 25 \mathrm{kDa}$} & Species: $\mathrm{mAb} / \mathrm{lgG}$ subunits (Lc, Fd, \& Fc/2) \\
\hline & $\begin{array}{l}\text { Sample preparation: structure-specific } \\
\text { digestion (IdeS, KGP, papain, etc.) followed } \\
\text { by S-S bond reduction }\end{array}$ \\
\hline & $\begin{array}{l}\text { CQAs: limited sequencing, PTMs (oxidation, } \\
\text { glycation, etc.) }\end{array}$ \\
\hline & $\begin{array}{l}\text { Methods: middle-up mass measurements } \\
\text { (direct infusion and RPLC-MS); middle-down } \\
\text { analysis with HCD/ETD/UVPD, ... }\end{array}$ \\
\hline \multirow[t]{4}{*}{$<10 \mathrm{kDa}$} & Species: proteolityc mAb/lgG peptides \\
\hline & $\begin{array}{l}\text { Sample preparation: sequence specific and } \\
\text { non-specific enzymes (trypsin, chymotrypsin, } \\
\text { Lys-C, Sap9, etc.) }\end{array}$ \\
\hline & $\begin{array}{l}\text { CQAs: complete sequencing (all CDRs), all } \\
\text { PTMs (deamidation, oxidation, etc.) }\end{array}$ \\
\hline & $\begin{array}{l}\text { Methods: RPLC-MS (extended) bottom-up } \\
\text { with HCD/ETD/UVPD, ... }\end{array}$ \\
\hline
\end{tabular}

Fig. 2. Molecular weight-organized overview of the MS-based approaches to the structural analysis of mAbs/lgGs, ADCs/AOCs, and other antibody-like molecules, including multi-specific antibodies Fc-fusion proteins. The acronyms and abbreviations are as follows. mAb: monoclonal antibody; IgG: immunoglobulin G; ADC: antibody-drug conjugate; AOC: antibody-oligonucleotide conjugate; CQA: critical quality attribute; DAR: drug-to-antibody ratio; RPLC: reversed-phase liquid chromatography; SEC: size exclusion chromatography; MS: mass spectrometry; HCD: higher-energy collision-induced dissociation; ETD: electron transfer dissociation; UVPD: ultraviolet photodissociation; CDR: complementarity determining region; PTM: post-translational modification; Lc: light chain; and Hc: heavy chain. in the sample preparation area have delivered novel, highly-specific enzymes, particularly for mAbs processing, e.g., hinge-region specific enzymes, IdeS (immunoglobulin-degrading enzyme of Streptococcus pyogenes) and KGP (lysine gingipain protease from Porphyromonas gingivalis), as recapitulated in Fig. 1. ${ }^{[25]}$

We were fortunate to integrate early on the MS and MS/MS instrumentation advances with the structural specificity provided by the novel enzymes and realize the benefits for mAb analysis. From our first steps in the analysis of mAbs a decade ago, we have promoted the use of both MS and MS/MS approaches that preserve as close as possible the precursor protein structure in the intact form. Thus, our focus has been, and remains, on the topdown view of the mAbs, including intact mass measurements, top-down, middle-up, and middle-down MS approaches.[26] One of the principal advantages of the top-down method compared with the more widely employed bottom-up approaches is its intrinsic ability to identify and characterize proteins at the proteoform level. ${ }^{[2,27]}$ Here, we briefly overview selected advances in both the methods and the techniques for MS-based structural analysis of mAbs pioneered by our groups, first at the Biomolecular Mass Spectrometry Laboratory at EPFL and later at Spectroswiss (Table 1). We believe these advances have helped to shape the landscape of MS-based structural analysis of mAbs into its current state (Fig. 2). We will briefly describe the development route of these methods and complement this retrospective with our current considerations for the MS-based characterization of mAbs/ IgGs and ADCs.

\section{Top-down ETD TOF MS of mAbs: Initial Implementation}

Historically, our decade-long journey in the field of MSbased mAb analysis started in 2010 with the pioneering experimental work on top-down analysis of whole mAbs (a human IgG1 and a murine IgG1) using ETD on a high-resolution quadrupole time-of-flight (TOF) MS instrument (MaXis $^{\mathrm{TM}}$ qTOF MS). This accomplishment, achieved in collaboration with Bruker Daltonics (Bremen, Germany), was revolutionary for its time due to the technical challenges of isolating and fragmenting large, $\sim 150 \mathrm{kDa}, \mathrm{mAb}$ species and resolving their many product ions on a TOF MS platform. The resolution performance of the TOF MS instrument employed in our work was lower than that potentially offered by Fourier transform mass spectrometry (FTMS), [28] but it was nevertheless exceptional for that time and varied from about 50000 for relatively sparse to about 30000 for the highly congested product ion regions. The results were published in 2011 (Table 1). [29]

Prior to this work, the analytically useful top-down MS on proteins of about this size was reported only using highresolution FTMS. A particular example is the top-down MS analysis of the linear formylglycinamide ribonucleotide amidotransferase (PurL) of about $143 \mathrm{kDa}$, performed without precursor ion selection, in the ion source region (post-skimmer dissociation) of an FTMS instrument, reported by McLafferty and co-workers in 2006. ${ }^{[30]}$ Shortly after, in 2009, Bondarenko and co-workers reported on the use of a similar approach, insource CID without precursor ion isolation, for the analysis of an intact $\mathrm{mAb}$ using the newly launched high-resolution instrument, a hybrid linear ion trap quadrupole (LTQ) Orbitrap ${ }^{\mathrm{TM}} \cdot{ }^{[28]}$ Another pioneering top-down MS analysis of large proteins, preceding our $\mathrm{mAb}$ work, was reported by Ge and co-workers also in 2009 , who managed to characterize the $\sim 142 \mathrm{kDa}$ protein $\mathrm{cMyBP}-\mathrm{C}$ and its truncated $\triangle \mathrm{C} 0-\mathrm{C} 1$ proteoform $(\sim 115$ $\mathrm{kDa}$ ) using CID and/or ECD applied to selected precursor ion charge states. ${ }^{[31]}$ The latter is perhaps the first top-down MS application that involved selected precursor ion isolation to proteins (not mAbs) above $140 \mathrm{kDa}$. It should be noted that the first attempt to analyze an intact $\mathrm{mAb}$ with the in-source CID was re- 
Table 1. An overview of selected advances in mass spectrometry-based mAb structural analysis developed by our groups.

\begin{tabular}{|c|c|c|}
\hline Year & Method / Advance & Ref. \\
\hline 2011 & $\begin{array}{l}\text { Top-down MS of intact mAbs with ETD on a TOF MS: first application of electron-assisted } \\
\text { radical dissociation reaction (ETD) to fragment intact mAbs }\end{array}$ & Tsybin et al. ${ }^{[29]}$ \\
\hline 2012 & $\begin{array}{l}\text { Top-down MS of intact mAbs (isotype 1) with ETD on an Orbitrap FTMS: first application of } \\
\text { electron-assisted radical dissociation reaction to fragment intact mAbs on an FTMS instrument }\end{array}$ & Fornelli et al. ${ }^{[35]}$ \\
\hline 2014 & $\begin{array}{l}\text { Middle-down MS of mAbs using IdeS enzyme ( } 25 \mathrm{kDa} \text { subunits) for improved sequencing and } \\
\text { PTMs analysis: first use of ETD in a combination with IdeS }\end{array}$ & Fornelli et al. ${ }^{[44]}$ \\
\hline 2014 & $\begin{array}{l}\text { Extended bottom-up MS of mAbs using Sap9 enzyme: up to } 100 \% \text { sequence coverage in a } \\
\text { single LC-MS/MS experiment with a novel enzyme, Sap9 }\end{array}$ & Srzentić et al..$^{[52]}$ \\
\hline 2017 & $\begin{array}{l}\text { Top-down analysis of isotypes } 1 \text { and } 2 \mathrm{mAbs} \text { with Orbitrap FTMS: first application of ETD to } \\
\text { intact } \mathrm{mAbs} \text { of isotype } 2 \text {, further method improvement }\end{array}$ & Fornelli et al. ${ }^{[41]}$ \\
\hline 2018 & $\begin{array}{l}\text { Multiplexed middle-down MS of mAbs using KGP enzyme ( } 50 \mathrm{kDa} \text { subunits): first } \\
\text { demonstration of light and heavy chain pairing via MS/MS of mAb subunits }\end{array}$ & Srzentić et al. ${ }^{[45]}$ \\
\hline 2021 & $\begin{array}{l}\text { Proteoform peak integration for intact mass analysis of antibody-drug conjugates: a novel } \\
\text { approach to drug-to-antibody ratio estimation for FTMS }\end{array}$ & Nagornov et al..$^{[46}$ \\
\hline
\end{tabular}

ported well before the FTMS work. Already in 1993, Feng and Konishi reported on this achievement using a triple-quadrupole mass spectrometer. Although the low-resolution performance of the employed MS platform significantly limited the analytical outcome, that original implementation confirmed that intact mAbs can be fragmented with CID in the gas phase. ${ }^{[77]}$

In our TOF MS work, ETD was chosen to replace collisioninduced ion activation and dissociation because of its propensity to more evenly cleave the peptide backbone, and its potential capability to rupture disulfide bridges (Fig. 3). ${ }^{[19]}$ An efficient implementation of ECD on a TOF MS platform was not available at that time, whereas currently there are several very potent ECD-enabled TOF MS configurations. [17h,32] The original topdown ETD results delivered $14 \%$ and $21 \%$ sequence coverage for the human and the murine mAb, respectively. Importantly, product ions were detected from the CDR regions, specifically the functionally critical CDR3 region. Overall, this advance was enabled by the ability of a novel high-resolution mass spectrometer to efficiently transfer heavy precursor ions and many product ions to the mass analyzer and to decipher a complex pattern of product ions. In addition, the already developed initial data analysis methods, including a high-resolution product ion deconvolution algorithm, facilitated analysis of the convoluted MS/MS datasets. ${ }^{[17 \mathrm{~g}]}$

The original implementation of the top-down ETD approach on the TOF MS platform allowed the formulation of the experimental design features and top-down data characteristics that are still actual today (Table 2). For example, the employed TOF MS instrument design entailed front-end injection of both multiply charged cations and radical azuline anions, with ETD reaction taking place in a collision chamber followed by an 'ion cooler' quadrupole. The latter was used to maximize the transfer efficiency of product ions to the TOF mass analyzer and their detection. ${ }^{[17 \mathrm{~g}]}$ Notably, a related approach to release product ions into the TOF mass analyzer has recently been shown to be instrumental in achieving the enhanced performance of ECD TOF MS implementation. ${ }^{[17 \mathrm{~h}]}$ For improving the ETD efficiency, the $100 \mathrm{~m} / \mathrm{z}$ isolation window included three charge states of the $\mathrm{mAb}$ precursor ions, centered around the 50+ charge state. The precursor ion accumulation time was increased compared to bottomup experiments, up to $800 \mathrm{~ms}$, and ETD duration (i.e., ion-ion interaction time) was typically set to $60 \mathrm{~ms}$. ETD product ion mass spectra, acquired with a broad, $100-5000, \mathrm{~m} / \mathrm{z}$ window, were dominated by the undissociated charge-reduced species (electron transfer without dissociation, or ETnoD, products), with multiplycharged product ions having a much lower intensity. The TOF
MS-specific baseline increase around the charge-reduced species is present also on the recently released high-performance ECD TOF MS configuration reported by Baba and co-workers, as exemplified by their top-down ECD analysis of bovine serum albumin. ${ }^{[17 \mathrm{~h}]}$

Depending on the analyzed $\mathrm{mAb}$, two different distributions in the product ion populations were observed in our work: a bimodal distribution characterized the murine mAb (Fig. 3, top panel), whereas the human $\mathrm{mAb}$ produced a single Gaussian-like product ion population (Fig. 3, middle panel). ${ }^{[29]}$ This difference was attributed to the gas-phase structural differences between the two mAbs. It was reflected on the fragmentation patterns identified for the two mAbs, with the N-terminus of both Lc and Hc highly sequenced in the case of the murine mAb (with this explaining the low $\mathrm{m} / \mathrm{z}$ product ion population) and with only three cleavage sites identified for the human mAb. Two main facts were associated with the fragmentation results for the two mAbs. First, they both showed that the primary sequence is not affected homogeneously by ETD fragmentation, but exhibited a pronounced sequencing along the loops interconnecting mAb domains, with the disulfide bond-protected areas and the central portion of the Hc remaining instead poorly characterized. Moreover, several fragmentation hot spots (preferred cleavage sites) were identified (Fig. 1, right panel, and Fig. 3, middle panel). The preference toward specific backbone cleavage sites is directed, presumably, by protein higherorder structure. ECD/ETD cleavage preference around basic amino acids, e.g., Lys, is known to be high and can be particularly strong if these amino acids are charged (protonated). ${ }^{[33]}$ Secondly, they both presented several backbone cleavage sites from disulfide bond-protected areas, confirming the capability of ETD of cleaving disulfide bonds in large proteins. These particular characteristics of intact mAb's fragmentation patterns in ETD MS/MS revealed by the original work, including the preferential fragmentation channels, are currently being exploited for novel method development in mAbs analysis, for example, for directing the de novo sequencing and $\mathrm{MS}^{\mathrm{n}}$ workflows. ${ }^{[34]}$

\section{Top-down ETD Orbitrap FTMS for mAbs: First Implementation}

To enhance the method's performance by further increasing the mass spectral resolution, we partnered with the Orbitrap development team at Thermo Fisher Scientific (Bremen, Germany). Prior to this work, Orbitraps were employed only for intact mass measurements of mAbs, in-source CID of whole mAbs, and CID-based middle-down analysis of mAbs, namely CID MS/MS of Lc and Hc subunits. ${ }^{[28]}$ To perform the initial top-down Orbitrap implementation, 

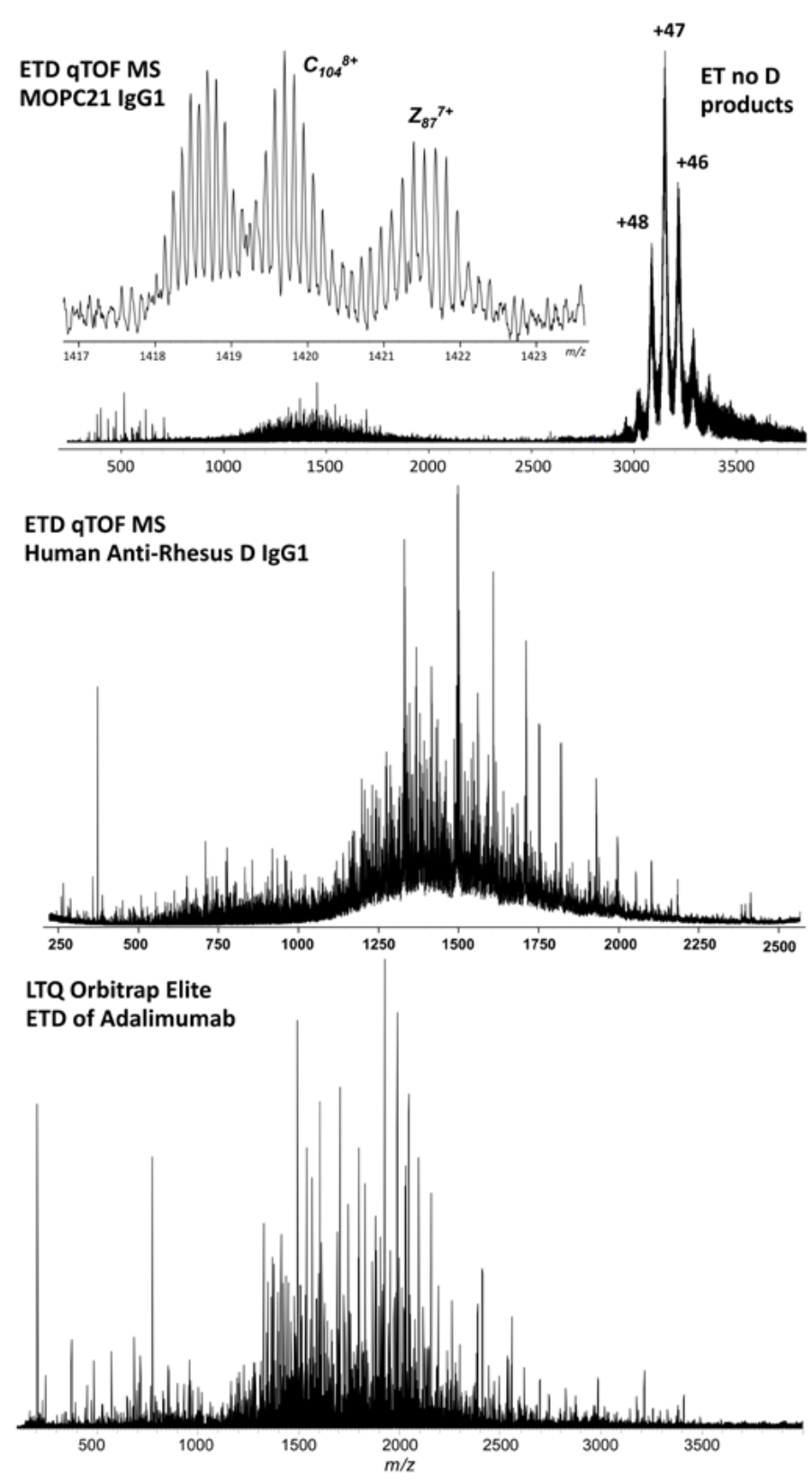

Fig. 3. Examples of top-down ETD mass spectra of mAbs. (Top panel) shows a tandem mass spectrum of MOPC21 mAb (a murine lgG1), acquired on a high resolution TOF MS (maXis ${ }^{\mathrm{TM}}$ ETD from Bruker Daltonics). The isolated precursor ions included charge states $+49,+50$ and +51 . The inset shows an expanded view of a complex product ion pattern demonstrating a high-resolution performance enabling assignment of multiply charged product ions. (Middle panel) shows application of the same qTOF MS set-up to the analysis of a human mAb, an antirhesus D IgG1. The same isolation window encompassing charge states 49-51 was employed. (Bottom panel) shows top-down analysis of a biotherapeutic mAb, Humira (adalimumab), performed with a hybrid LTQ Orbitrap $^{\mathrm{TM}}$ Elite $^{\mathrm{TM}}$ equipped with ETD. Precursor ion isolation window $2900+/-300 \mathrm{~m} / \mathrm{z}$, ETD duration 10 and $25 \mathrm{~ms}, 1000$ summed scans, target number of charges 1e6. Figures are adapted from Tsybin et al.,, ${ }^{[2]}$ and Fornelli et al. ${ }^{[41]}$

we employed a therapeutic IgG1 mAb - adalimumab (Humira). The experiments were conducted with a hybrid LTQ Orbitrap instrument $\left(\right.$ Orbitrap $^{\mathrm{TM}}$ Velos Pro $^{\mathrm{TM}}$ ). The Orbitrap mass analyzer was operating with a resolution of 100000 at $400 \mathrm{~m} / \mathrm{z}$, which is almost double that sustained by the high-resolution TOF MS described above. The result of this pioneering Orbitrap ETD-based top-down analysis of mAbs was published in 2012 (Table 1). ${ }^{[35]}$
The first Orbitrap application to a whole $\mathrm{mAb}$ analysis with ETD MS/MS relied, first of all, on the benefits of higher resolution offered by FTMS compared to even the state-of-the-art in TOF MS technology. The increased resolution in FTMS was shown to be beneficial for dealing with product ion isotopic envelopes overlapping with the pronounced baseline around the abundant precursor and charge reduced ions in TOF mass spectra (Fig. 3, bottom panel and Fig. 4).[17g,h] The selection of the optimum ETD ion-ion reaction times was performed based on the variation of product ion yield and abundance of ETnoD species (Fig. 4). In addition, we employed the fundamental ability of FTMS instruments to increase the sensitivity of peak detection in mass spectra by averaging the unreduced data - the time-domain transients. ${ }^{[36]}$ It is known that averaging $N$ time-domain transients increases the signal/noise ratio (SNR) of mass spectral peaks proportionally to the square root of $N .{ }^{[37]}$ The more common at that time direct infusion sample introduction would allow many time-domain transients to be aquired in a single experiment. ${ }^{[38]}$ However, direct infusion necessarily requires off-line $\mathrm{mAb}$ purification and desalting (Fig. 2). Therefore, to make the method more straightforward and minimize sample manipulation, we early on decided to develop $\mathrm{mAb}$ analysis methods based on LC online coupled with MS, or LC-MS. In the case of mAbs, due to sample availability and the targeted protein analysis approach, the number of time-domain transients can be increased substantially by performing several technical replicates of LC-MS/MS measurements.

In the Orbitrap-based method implementation, the electron transfer from fluoranthene radical anions to multiply-charged $\mathrm{mAb}$ cations was performed in the LTQ. ${ }^{[35]}$ The ion-ion interaction times were shortened compared to TOF MS implementation, and the number of reacting species (number of anions and number of cations) was kept nearly constant by the automatic gain control (AGC) function implemented in the LTQ. Furthermore, similarly to the TOF MS experiments, multiple charge states of the precursor ions were selected by isolating precursor ions with a $100 \mathrm{~m} / \mathrm{z}$ window (equivalent to three charge states, from $53+$ to $55+$ ) or a $600 \mathrm{~m} / z$ window (including charge states from $47+$ to $57+$ ) (Table 2). The mass spectra were recorded between 200 and $4000 \mathrm{~m} / \mathrm{z}$, and represented in magnitude mode FT $(\mathrm{mFT})$. As a reminder, the mass spectral representation in the 'historic' mFT mode available at that time provided about twotimes lower resolution for the same ion detection period (time-domain transient length) compared to the current industry-standard enhanced FT, or eFT, mass spectral representation in Orbitrap FTMS. [39]

An important characteristic of the Orbitrap-based experiment design was the acquisition of tandem mass spectra with two different ETD reaction durations, 10 and 25 ms. ${ }^{[35]}$ The shorter ETD duration produced tandem mass spectra with product ions centered around $\mathrm{m} / \mathrm{z}, 1600$, whereas longer duration resulted in their shift towards higher $m / z$. Acquiring time-domain transients of ETD product ions allowed the combination of results of 10 and $25 \mathrm{~ms}$ ETD MS/MS experiments in a single mass spectrum (Fig. 3, bottom panel). The undissociated charge-reduced (ETnoD) species at the chosen reaction times, 10 and $25 \mathrm{~ms}$, dominated Orbitrap ETD MS/MS spectra less compared with the TOF MS/MS. The final results showed a substantial improvement in sequence coverage in relation to the previous TOF ETD MS/MS study, with $24 \%$ sequence coverage with the $100 \mathrm{~m} / z$ isolation window and $27 \%$ coverage with the $600 \mathrm{~m} / z$ window, considering only $c^{-}, z^{-}$, and $y$-type product ions. Overall, the combination of the two sets of experiments (for a total of about 13000 time-domain transients - obtained by acquisition of many LC-MS/MS technical replicates) led to a record $32.7 \%$ sequence coverage (Table 2 ). A careful analysis of the produced fragmentation map revealed that experiments involving different precursors resulted in high coverage of slightly different areas, probably due to the different arrangements of the protonation sites. Globally, the highly sequenced regions remained unvaried compared to the previous top-down TOF ETD MS/MS work. 
Table 2. An overview of instrument-specific selected characteristics of the mass spectrometry-based mAb structural analysis methods developed by our groups.

\section{Instrument}

MaXis ETD

q TOF MS

LTQ Orbitrap Velos Pro

LTQ Orbitrap Elite

Q Exactive HF BioPharma

\section{Method Characteristics}

Top-down analysis of a murine and a human $\operatorname{IgG} 1 \mathrm{~s}$

Direct infusion on a high-resolution instrument: $30000-50000 \mathrm{~m} / \Delta \mathrm{m}$

Wide isolation windows for precursors $(100 \mathrm{~m} / \mathrm{z}$ isolates 3 charge states)

Improved product ion transmission and detection: 'ion cooler' quadrupole

Abundant ETnoD products dominate ETD mass spectra

Baseline increase around ETnoD peaks is pronounced (low resolution)

Extensive fragmentation outside $\mathrm{S}-\mathrm{S}$ bond protected regions

Preferential cleavage sites suggest $\mathrm{mAb}$ structure-cleavage relationships

Sequence coverage: up to $20 \%$

Top-down analysis of a biotherapeutic IgG1 - Humira (adalimumab)

On-line LC-MS/MS for sample purification, denaturing conditions

D30 Orbitrap, mFT mass spectra: higher resolution (100 000 at $\mathrm{m} / \mathrm{z} 400$ )

Wide isolation windows: $100 \mathrm{~m} / \mathrm{z}$ and $600 \mathrm{~m} / \mathrm{z}$ (up to 10 charge states)

The undissociated ETnoD species are less pronounced

Baseline increase around ETnoD products is resolved (high resolution)

Averaging the time-domain transients from technical LC-MS replicates

Dual ETD reaction duration: $10 \mathrm{~ms}$ and $25 \mathrm{~ms}$ for increased coverage

Sequence coverage $\sim 30 \%$, but long experiment

Top-down analysis of $\mathrm{IgG} 2$ and $\mathrm{IgG} 4$, diverse $\mathrm{IgG} 1$ biotherapeutic mAbs D20 Orbitrap, eFT mass spectra - faster scanning, enhanced resolution Method optimization: 'HCD trapping' and 'protein mode' pressure regime Multiple ETD reaction times provide complementary product ions Sequence coverage in top-down remains at $\sim 30 \%$, but faster acquisition Middle-up and middle-down approaches development: mAb subunits Sequence coverage in middle-down: up to $60 \%$ (total mAb sequencing) Light and heavy chain pairing: top-down analysis of $F(a b)$ subunits Extended bottom-up analysis with Sap9: up to $100 \%$ sequence coverage

aFT mode mass spectra representation (higher resolution, sensitivity) Averaging aFT mass spectra or transients provides equal performance Proteoform integration approach improves ADC/AOC analysis of DARs Sliding window approach in LC-MS increases accuracy for DAR analysis

\section{Top-down ETD of mAbs on High-field Orbitrap FTMS}

The success of the initial intact mAb analysis with ETD on TOF and regular Orbitrap mass spectrometers in Bremen resulted in the technology transfer to our laboratory at EPFL in Lausanne (Fig. 3, bottom panel). Due to our original expertise and interest in FTMS and the already available ion cyclotron resonance (ICR) technology (a 12 T LTQ FT-ICR MS instrument) in the laboratory, we continued to perform further method development and applications on a hybrid LTQ Orbitrap ${ }^{\mathrm{TM}}$ Elite $^{\mathrm{TM}}$ platform. The custom-built Orbitrap instrument installed in our laboratory uniquely at that time allowed us to acquire even higher resolution mass spectra (including $3 \mathrm{~s}$ or even longer transients delivering a 1 million resolution at $\mathrm{m} / \mathrm{z}, 400$ and above) and provided access to the time-domain transients from the built-in data acquisition system. ${ }^{[40]}$ Our follow-up work on the top-down analysis of mAbs on this powerful platform improved the method performance (Tables 1 and 2).[41] The realized improvements allowed to substantially reduce the number of averaged transients and, ultimately, the required experimental time. These were achieved by an enhanced efficiency to select higher charge state precursor ions, increased instrument data acquisition rate (high-field, compact Orbitrap mass analyzer, D20, and eFT mass spectra) compared to the LTQ Orbitrap $^{\mathrm{TM}}$ Velos Pro ${ }^{\mathrm{TM}}$ configuration, ${ }^{[37,39]}$ improved ion manipulation including an extension of the ion path prior to $\mathrm{C}$-trap focusing and injection to the Orbitrap (now known as 'HCD trapping'), and (manual) reduction of the collision gas pressure in the HCD cell/C-trap to lower the pressure in the ultra-high-vacuum region where the Orbitrap mass analyzer is located. These procedures are now automated and represent the foundation of the 'protein mode' available on modern Orbitrap platforms. ${ }^{[42]}$ In addition, we confirmed that ETD reactions performed with multiple reaction times lead to complementary fragmentation patterns. This approach has been recently taken further by jointly considering data obtained with different MS/MS techniques, such as CID and ETD, to benefit sequence coverage. ${ }^{[12 b, 22 b]}$

Overall, including sequence-specific product ions from individual experiments performed with different reaction times reduced the number of averaged transients necessary for achieving $\sim 30 \%$ sequence coverage to approximately 6000 . Nevertheless, the '30\%-barrier' was not greatly overcome, suggesting that other, complementary, approaches are needed.

\section{Middle-down ETD Orbitrap FTMS of mAbs}

Despite the improvements in the top-down approach performance enabled by better instrumentation, optimization of the experimental procedures, and advances in data processing and analysis, the final sequence coverage outcome remained restricted, presumably due to structural constraints, such as $\mathrm{S}-\mathrm{S}$ bonds and the large size of subunits forming a mAb. Therefore, in parallel with the 

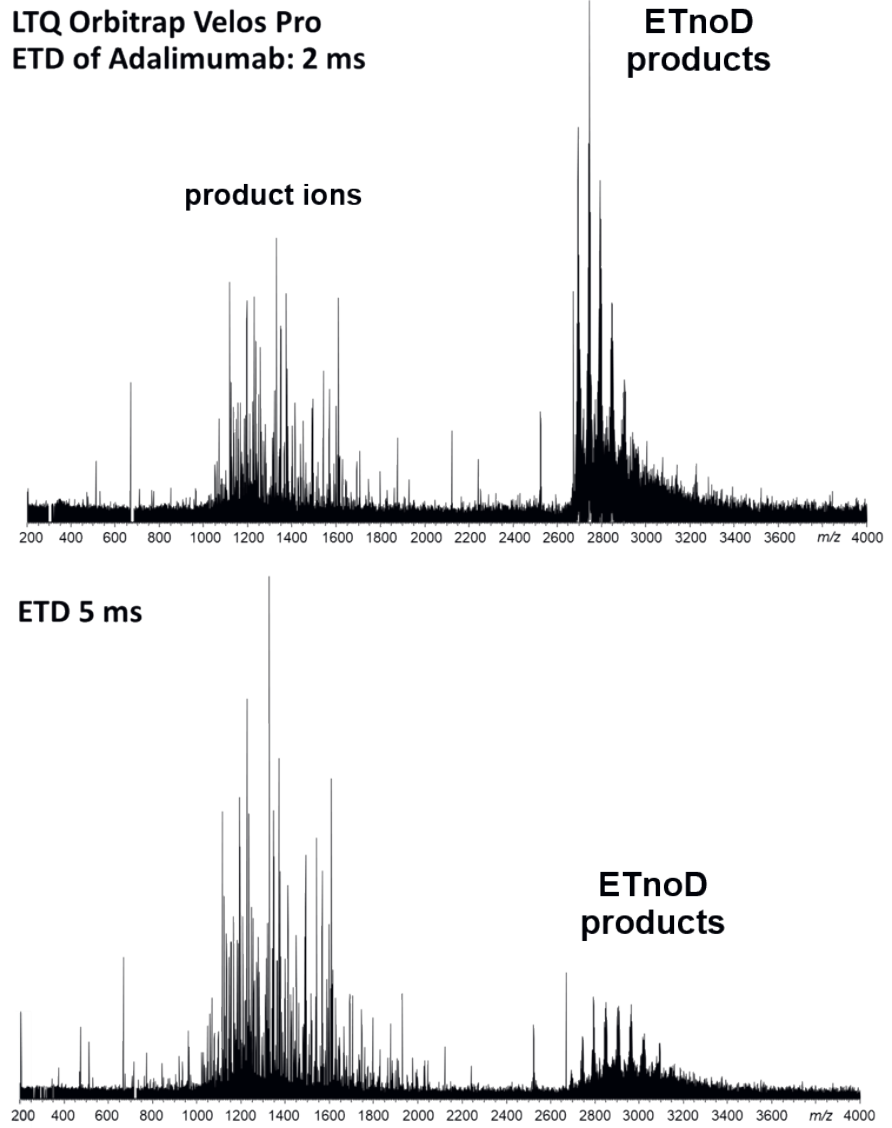

ETD $50 \mathrm{~ms}$

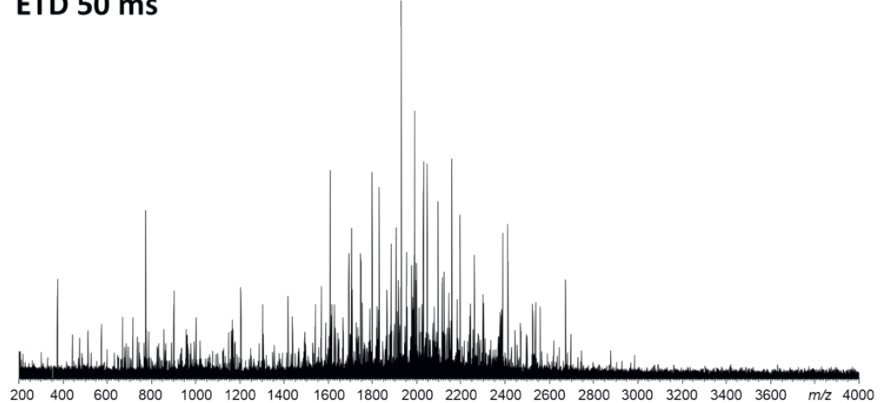

Fig. 4. Influence of ETD ion-ion reaction time on the top-down mass spectra composition in the analysis of a mAb. Shown are the results of ETD top-down MS of adalimumab (Humira) analyzed with a hybrid LTQ Orbitrap $^{\mathrm{TM}}$ Velos Pro ${ }^{\mathrm{TM}}$ for different ETD reaction times: 2 ms (top panel), $5 \mathrm{~ms}$ (middle panel, and $50 \mathrm{~ms}$ (bottom panel). Precursor ion isolation at $2750+/-50 \mathrm{~m} / \mathrm{z}$. The experimental details are described in Fornelli et al. ${ }^{[35]}$

top-down approach, we started to develop the middle-down methods (Fig. 2). These are defined as the protein structural analysis approaches based on the gas-phase fragmentation of precursor ions of parts (e.g., mAb subunits) of a protein (Fig. 1). ${ }^{[26]}$ To qualify for the middle-down definition, these protein parts should be relatively large, 3-5 fold greater than the typical enzymatically-derived peptides analyzed in bottom-up MS approaches, or represent a structural unit of a precursor protein. In the case of mAbs, which are characterized by a multi-chain quaternary structure, functionally and/ or structurally distinct subunits can be generated by disulfide bond reduction or by enzymatically-assisted digestion in the hinge region (Fig. 1). ${ }^{[12 b, 28]}$ If the disulfide bond reduction is performed under denaturing conditions, in the presence of chaotropic agents such as urea or guanidine- $\mathrm{HCl}$, all disulfide bonds could be reduced, yielding separate $\mathrm{Lc}, \sim 25 \mathrm{kDa}$, and $\mathrm{Hc}, \sim 50 \mathrm{kDa}$ (Figs 1 and 2). The other types of mAb subunits, e.g., $\mathrm{F}(\mathrm{ab})$ and $\mathrm{Fc}$, are produced by limited proteolysis of mAbs using non-denaturing conditions.
We selected a mAb structure-specific bacterial cysteine protease IdeS for our implementation of a middle-down approach. The specificity of IdeS to $\mathrm{mAb}$ digestion is in the unique cleavage site located just below the hinge region (Fig. 1).[25] This enzyme became commercial at about that time and demonstrated an interesting use in the middle-up applications to mAb analysis. ${ }^{[25,43]}$ The IdeS-derived $\mathrm{mAb}$ subunits after the digestion are the $100 \mathrm{kDa}$ $\mathrm{F}(\mathrm{ab})$ ', and the $50 \mathrm{kDa} \mathrm{Fc}$ subunits. Note, the prime ' notation is added to the subunit name to denote a presence of a hinge region in it. The Fc subunit is held together by non-covalent interactions and thus usually appears as two separated Fc/2 subunits of $\sim 25$ $\mathrm{kDa}$ each when denaturing conditions are used for LC-MS analysis (Fig. 1). The $\mathrm{S}-\mathrm{S}$ bonds that keep the $\mathrm{F}(\mathrm{ab})_{2}{ }_{2}$, subunit together may be efficiently reduced to yield two $\sim 25 \mathrm{kDa}$ subunits - namely $\mathrm{Lc}$ and $\mathrm{Fd}$, which is the N-terminal part of the Hc. Therefore, instead of a single $150 \mathrm{kDa} \mathrm{mAb}$, IdeS allowed to deal with three $\mathrm{mAb}$ subunits of $\sim 25 \mathrm{kDa}$ each (Lc, Fd', and Fc/2) (Fig. 1).

As a result, we combined the instrumentation and sample-preparation advances to develop a novel workflow for $\mathrm{mAb}$ structural analysis-theETDMS/MS-based middle-down approach(Fig. 2). ${ }^{[44]}$ The results obtained with this workflow improved significantly, by $2-3$-fold, the sequence coverage obtained by the top-down approach. In addition, the involved processes, such as the separation of the $25 \mathrm{kDa}$ species with an LC system and the subsequent ion formation, transfer, and detection with an Orbitrap mass spectrometer, were substantially easier to perform and optimize compared with the whole $\mathrm{mAb}$ analysis. It is thus not surprising that this workflow is gaining an increasing interest in biopharma analytical laboratories and constitutes a part of commercial software solutions.

Compared to the whole $\mathrm{mAb}$, the $\sim 100 \mathrm{kDa} F(\mathrm{ab})_{2}$ subunit generated by IdeS digestion is structurally-simplified as it lacks the heterogeneous $\mathrm{Fc}$ subunit that carries the canonical N-linked glycans. In addition, it is composed of an Lc and an Fd subunit, the known pairing of which may help identify a whole mAb due to the presence of all six CDRs on them. Nevertheless, the attempted middledown analysis of the $\mathrm{F}(\mathrm{ab})_{2}$, subunit did not substantially improve the final sequence coverage compared with top-down analysis.

To further facilitate the middle-down analysis application for the chain pairing, we employed another structure-specific enzyme, KGP (commercially available from Genovis as GingisKHAN). [45] This cysteine protease is particularly efficient towards the IgG1 class, and it cleaves in a highly specific and reproducible manner at the ...KSCK-THTCPPCP... bond above the hinge region (Fig. 1). Therefore, the resulting digestion products are $\sim 50 \mathrm{kDa}$ subunits - two F(ab) subunits and an Fc subunit (held together by covalent intermolecular $\mathrm{S}-\mathrm{S}$ bonds in the hinge region). The $\mathrm{F}(\mathrm{ab})$ subunit is thus the smallest part of a mAb that may provide the Lc and $\mathrm{Hc}$ chain pairing information. We realized the corresponding proof-of-concept experiment using trastuzumab (Herceptin) as a reference $\mathrm{mAb}$ (Table 1). ${ }^{[45]}$ The experiments were performed on an LTQ Orbitrap ${ }^{\mathrm{TM}}$ Elite $^{\mathrm{TM}}$ equipped with ETD MS/MS and a Q Exactive ${ }^{\mathrm{TM}} \mathrm{HF}$ with the BioPharma option equipped with higher-energy collision-induced dissociation (HCD) MS/MS. Once again, the sensitivity enhancement via data averaging from technical replicates demonstrated its enabling power to enhance confidence in both ETD and HCD product ion annotation and increased sequence coverage. The reported results were the first ones to represent Orbitrap mass spectra in a true absorption FT mode (aFT). ${ }^{45]}$ The latter advance was due to the phase artifact-free acquisition of time-domain transients from an Orbitrap using a high-performance data acquisition system, FTMS Booster X2 (Spectroswiss). ${ }^{[46]}$ In contrast to the mFT and eFT mass spectra, the aFT mass spectra preserve full information on the ion signals contained in the time-domain transients: frequency, intensity, and phase. Fundamentally, only the time-domain transients and the aFT mass spectra represent the unreduced data types in FTMS. Using either of them would yield equal information upon data averaging, retiring the concept of microscans. Overall, 
the use of the unreduced data maximizes the information output and increases confidence in FTMS data. ${ }^{[47]}$

Other common proteases suitable for middle-down approaches include papain, pepsin, ficin, and endoprotease Lys-C. ${ }^{[48]}$ However, compared to IdeS and KGP, optimizing sample preparation using these proteases can be more laborious, the yield is generally more heterogeneous, and some of them may suffer low specificity. Nevertheless, our original proof-of-concept experiments on pairing light and heavy chains of mAbs, prior to the use of KGP, were performed with papain on a single mAb and simple mAb cocktails; see below.

A formidable challenge of extending the chain pairing approach from a single $\mathrm{mAb}$ to simple cocktails and more complex mixtures of mAbs can be appreciated already from the analysis of a mixture of three therapeutic mAbs (Fig. 5). The first analyzed reference mixture consisted of three mAbs: adalimumab, trastuzumab, and natalizumab. After papain digestion, the mAb subunit mixture was directly analyzed by LC-MS/MS on a hybrid LTQ Orbitrap ${ }^{\mathrm{TM}}$ Elite $^{\mathrm{TM}}$ instrument. Fig. 5 shows the total ion current (TIC) chromatogram of the mixture, demonstrating separation of $\sim 50 \mathrm{kDa} \mathrm{Fc}$ and F(ab) subunits. Expectedly, the Fc subunits of all three mAbs co-eluted as they share nearly identical sequences. The Fc subunits contain glycosylation and other structural features important for effector functions, interaction with Fc receptors, and stability. Still, they provide no information about Lc and Hc pairing as they originate solely from the heavy chains.

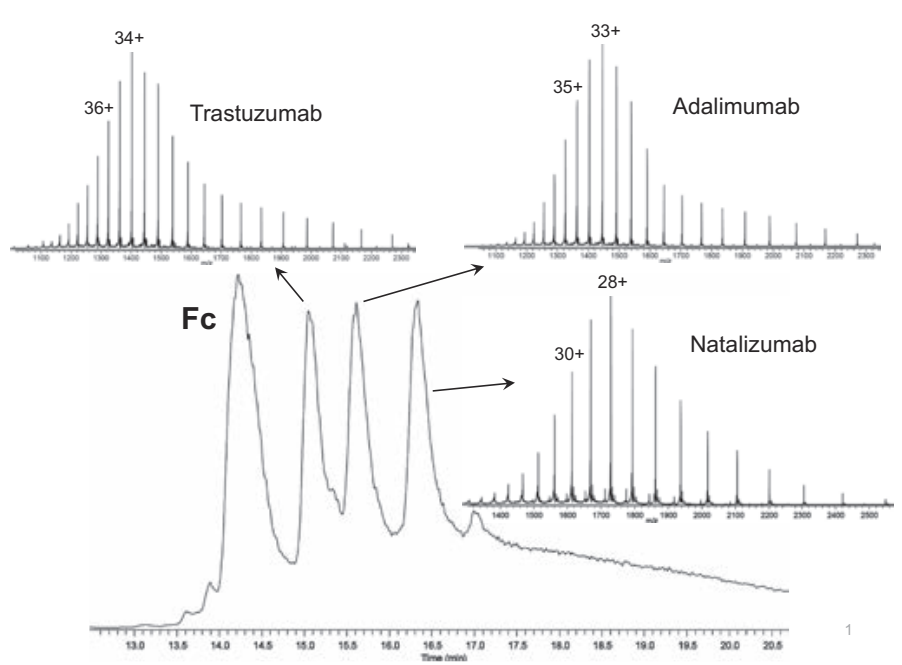

Fig. 5. An example of a middle-up approach application to mAb analysis. The main panel shows a TIC chromatogram of separated $50 \mathrm{kDa}$ $F(a b)$ subunits of three mAbs from a mixture digested with papain: IgG1 trastuzumab, IgG1 adalimumab, and IgG4 natalizumab. Additional panels show broadband LTQ OrbitrapTM EliteTM mass spectra of the charge state envelopes of the corresponding $F(a b)$ subunits of these mAbs. The precursor ion isolation windows for the subsequent ETD MS/ MS are centered around charge states 34-36, 33-35, and 28-30 for trastuzumab, adalimumab, and natalizumab, respectively.

The three $\mathrm{F}(\mathrm{ab})$ subunits originating from the three IgGs were separated by reversed-phase liquid chromatography (RPLC) under denaturing conditions, ${ }^{[18 a]}$ (Fig. 5). Maintaining the LC column at a constant temperature $\left(e . g ., 65^{\circ} \mathrm{C}\right)$ ensured the high elution reproducibility which is necessary for subsequent elution time scheduled ETD-based fragmentation. Nevertheless, baseline separation of the $F(a b)$ subunits of the three mAbs was not achieved. This result is not surprising, taking into account the close hydrophobicity values that are characteristic for such large mAbs subunits (which include highly conserved constant regions). Despite of the absence of the baseline separation, precur- sor ion isolation using a wide isolation window (encompassing several charge states) was possible. The latter is beneficial to maximize ETD MS/MS efficiency and sequence coverage. The choice of precursor ion charge states is driven by the efficiency of ETD being proportional to precursor charge state. Therefore, we attempted to preferentially isolate higher charge states precursor ions that were abundant enough to ensure the ion injection (accumulation) times are compatible with the LC timescale. An example of a resulting ETD mass spectrum is shown in Fig. 6.

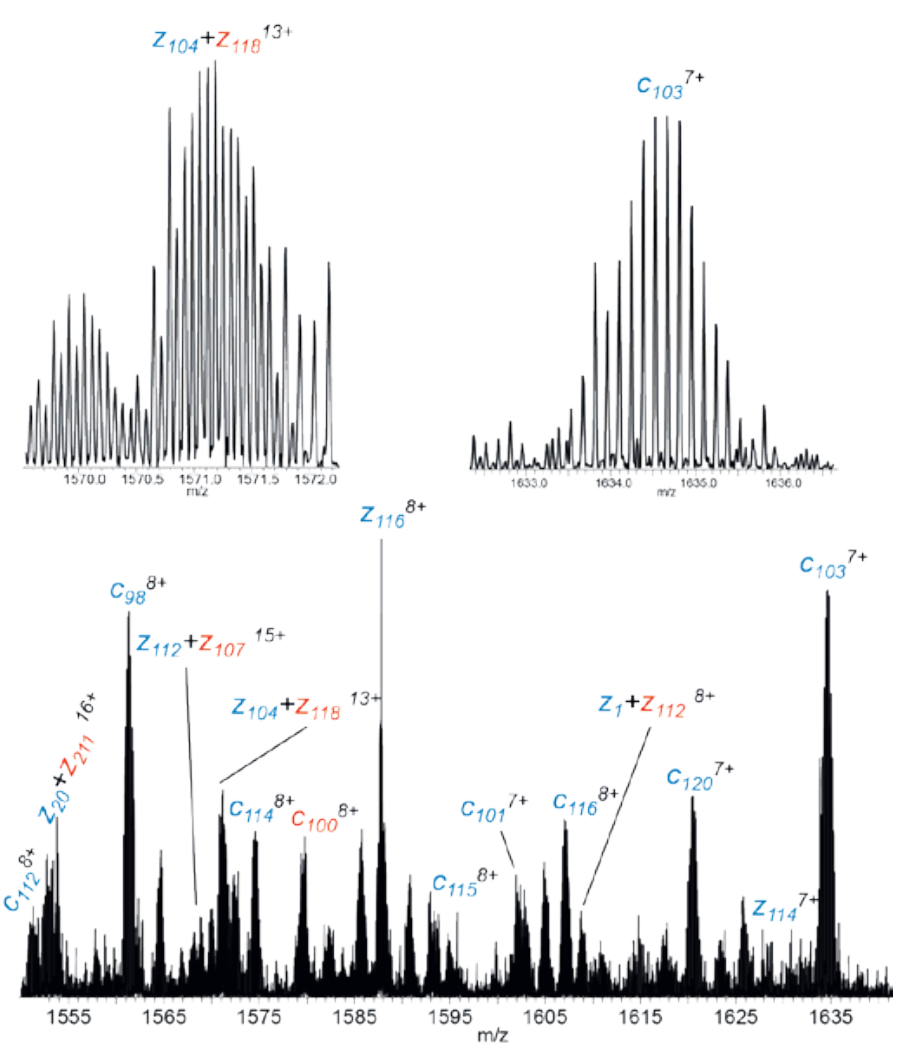

Fig. 6. Middle-down MS product ion complexity: an expanded view of ETD mass spectrum ( $\mathrm{m} / \mathrm{z} 1500-1640$ range) of Fab subunit of trastuzumab (generated with papain digestion, Fig. 5). Indicated are terminal $\mathrm{c}$-ions and disulfide bond linked $z-z$ ions. Insets show expanded views of isotopically resolved product ions identified as an interchain disulfide bond linked z104-z118 (+13 charge state) product ion (left) and the c103 (+7 charge state) terminal product ion (right). Product ions shown in blue belong to $\mathrm{Hc}$ and in red - to Lc.

If a co-elution of mAb subunits occurs, one can use narrow isolation windows for individual charge state isolation from each co-eluting species and obtain targeted MS/MS spectra that correspond to the particular $\mathrm{F}(\mathrm{ab})$ subunits. This procedure is challenging on an LC-MS peak elution profile as only a few timedomain transients can be acquired, which makes increasing the number of LC-MS/MS technical replicates beneficial to detect more product ions and increase SNR. Another alternative is to isolate several charge states for both $\mathrm{F}(\mathrm{ab}) \mathrm{s}$. A mixture of trastuzumab and rituximab, two mAbs that provide co-eluting $\mathrm{F}(\mathrm{ab})$ subunits in the RPLC-MS settings employed, were analyzed using a broad isolation window of $230 \mathrm{~m} / z$ to test if the quality of the $\mathrm{F}(\mathrm{ab})$ sequencing would be affected and if it would still be possible to identify the chain pairing-specific product ions connected by an inter-chain disulfide bond (Fig. 7). The product ion analysis results showed that the co-isolation of several charge states from both mAbs might preserve the outcome of the sequence coverage 
in data analyses performed with and without considering disulfide bond-linked product ions (data not shown). The CDR3 domains that are particularly important for unambiguous chain pairing were covered with several product ions in all cases. Moreover, rituximab was confirmed to present cyclization of glutamine to pyroglutamic acid on both chains. Overall, this result suggests that the sequencing and chain pairing determination can be possible even in the case of species co-elution, supporting the validity of the middle-down approach at the $\mathrm{F}(\mathrm{ab})$ level for mAb mixtures.

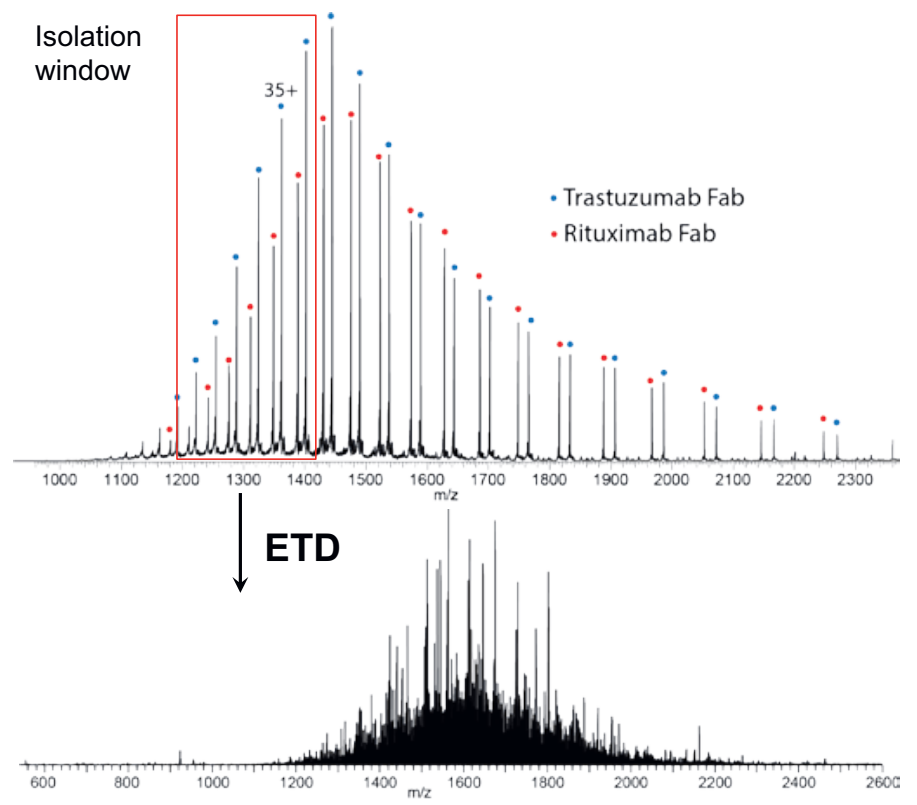

Fig. 7. An example of a middle-down approach application to mAb analysis. The figure shows a broadband LTQ OrbitrapTM EliteTM mass spectrum (top panel) and ETD tandem mass spectrum (bottom panel) of the co-eluting trastuzumab and rituximab $F(a b)$ subunits. The respective charge state envelopes are color-coded. The red rectangle shows the isolation window of $\sim 230 \mathrm{~m} / \mathrm{z}$ employed for ETD MS/MS. The isolation window contains five to six charge states of each $F(a b)$ subunit.

\section{Extended Bottom-up Mass Spectrometry of mAbs}

Comprehensive structural analysis of mAbs requires a $100 \%$ sequence coverage and identification of small, such as $\sim 1 \mathrm{Da}$, modifications. A common $\sim 1$ Da modification is asparagine (Asn) deamidation (loss of $\mathrm{NH}_{3}$ and gain of $\mathrm{H}_{2} \mathrm{O}$ ), which transforms Asn residues into aspartic (Asp) and iso-aspartic acid residues (isoAsp) via formation of a succinimide intermediate. ${ }^{[49]}$ To analyze such minor modifications, neither top-down nor middle-down approaches may be sufficient. Instead, a standard workflow would be based on the bottom-up approach. This approach is more commonly applied to the analysis of proteomes and is typically performed with trypsin as the enzyme of choice. Trypsin specificity is toward the basic amino acids - Lys and Arg. Due to the relatively high (about one out of 10 amino acids) occurrence of these amino acids in proteins, mAbs included, trypsin-derived peptides are typically lighter than $3 \mathrm{kDa}$. Nevertheless, even such significant sample simplification into relatively short tryptic peptides does not always provide a complete sequence coverage of mAbs. ${ }^{[12 \mathrm{~b}]}$ First, mAbs are known to carry an extended amino acid region without basic residues, which would result in the generation of very large peptides that may remain undetected with standard experimental settings. In addition, mAbs may have regions with many basic residues, which would result in the generation of very short peptides, composed of less than 5 amino acids, that may escape the detection. Furthermore, digestion reaction with trypsin usually involves steps performed at a high $\mathrm{pH}$ level. The latter is known to induce structural changes, such as inducing Asn deamidation. Therefore, enzymatic reactions at a lower $\mathrm{pH}$ level and providing a $100 \%$ sequence coverage were of interest at that time (presently, novel trypsin-based enzymatic digestion procedures may be performed at reduced $\mathrm{pH}$ conditions).

To address this need, we characterized a novel enzyme - the secreted aspartic protease Sap9 - and benchmarked its utility for $\mathrm{mAb}$ structural analysis. The original aim was to employ Sap9 for developing an alternative middle-down approach due to the enzyme's suggested dibasic (e.g., KK, RR, KR, and RK) amino acid cleavage specificity. ${ }^{[50]}$ However, our extensive protease characterization revealed its less strict amino acid preference. ${ }^{[51]}$ Nevertheless, the Sap9-based workflow fulfilled several requirements and resulted in a novel approach distinct from both middledown and bottom-up - extended bottom-up mass spectrometry (Table 1). ${ }^{[52]}$ Its application to diverse mAbs showed up to $100 \%$ sequence coverage and minimal introduction of artifacts, such as almost absent isomerization of Asp residues during digestion. Adding the term 'extended' was justified by the 2-fold larger size of the proteolytic peptides produced by Sap 9 compared to trypsin. The access to larger and, thus, more highly charged peptides was believed to be beneficial for high-resolution mass spectrometry and charge state-dependent ETD MS/MS.[50]

Finally, the Sap9-based extended bottom-up approach showed promise for rapid de novo sequencing of $\mathrm{mAbs}$, particularly using the algorithms originally developed for the top-down methodology. [53] Indeed, the current industry standard de novo sequencing of $\mathrm{mAbs}$ is based on bottom-up approaches that utilize several enzymes with complementary cleavage specificities. ${ }^{[54]}$ In the panel of these proteases, which usually includes 3-5 enzymes, trypsin is generally complemented by chymotrypsin and Lys-C. On the other hand, results obtained by the Sap9-based extended bottom-up workflow suggest that a single enzyme, Sap9, may replace the multi-enzyme approach or, at least, reduce the number of the required proteases.

\section{Intact and Middle-up Mass Measurements of mAbs and ADCs}

Intact mass measurements of mAbs, ADCs, and mAbs subunits are indispensable parts of top-down and middle-down workflows (Fig. 2). ${ }^{[10 a, 55]}$ Modern MS offers both low- and high-resolution capabilities for intact mass measurements, allowing mAb analysis at the isotopically-resolved and unresolved levels. ${ }^{[56]}$ The former strategy is widely applied for $\sim 25 \mathrm{kDa}$ mAb subunit analysis (middle-up approach), whereas the latter typically provides proteoform-resolved mass spectra for larger mAb subunits and intact $\mathrm{mAb}$ species, e.g., for glycosylation profiling. ${ }^{[57]}$ The lowresolution intact mass measurements provide average mass values and, advantageously, integrate ion signals from all the isotopes constituting a mAb molecule. ${ }^{[58]}$ That increases the SNR of the detected peaks by orders of magnitude.

Higher resolution enables the differentiation of $\mathrm{mAb}$ species with smaller mass differences. However, the extensive isotopic envelope of $\mathrm{mAbs}$ ( $\sim 25 \mathrm{Da}$ wide) limits the capabilities of intact mass measurements to resolve closely-spaced proteoforms (e.g., single oxidation of $+16 \mathrm{Da}$, or dehydration, $-18 \mathrm{Da}$ ) using the usually accessible and/or LC-compatible MS resolution performance. ${ }^{[46]}$ Therefore, similar to the case of middle-down approaches described above, to better distinguish the different $\mathrm{mAb}$ proteoforms, the mass analysis of structurally-significant $\mathrm{mAb}$ subunits, e.g., F(ab), F(ab), Hc, Lc, Fd, Fc, and Fc/2, can be preferred (Figs 1 and 2). In this regard, middle-up MS provides an extensive profile picture of the structural micro-variants of the molecule. ${ }^{[59]}$ Although middle-up MS assigns these modifications to a certain $25 \mathrm{kDa}$ subunit, it does not allow to attribute them to 
a specific amino acid. Therefore, the intact and middle-up mass measurements are generally complemented by the discussed (extended) bottom-up, middle-down, and top-down MS approaches (Fig. 2). ${ }^{[60]}$ The (extended) bottom-up approaches complete the intact/middle-up capabilities in the analysis of the tiniest modifications.

In regard to intact mass measurements, attempts to resolve proteoforms of complex mAb/ADC samples may result in highly congested mass spectra that may be challenging or impossible to rationalize. ${ }^{[18 \mathrm{a}, 61]}$ It is thus a common practice to deglycosylate $\mathrm{mAb} / \mathrm{ADC}$ samples prior to LC-MS (and LC-MS/MS) analysis, for example by using PNGase F (Peptide:N-glycosidase F) amidase (Fig. 2). ${ }^{[3,57]}$ To potentially avoid this additional sample preparation step, we recently demonstrated that integration of mass spectral peaks from several proteoforms provides ultra-lowresolution mass spectra that can be beneficial for the DAR analysis of complex ADC/AOC samples (Table 1 and Fig. 1). ${ }^{[46]}$

Briefly, the experimental measurements of an AOC sample (trastuzumab-oligonucleotide conjugate) were performed on a $\mathrm{Q}$ Exactive ${ }^{\mathrm{TM}} \mathrm{HF}^{\mathrm{TM}}$ mass spectrometer equipped with the BioPharma option. ${ }^{[46]}$ This allowed for ion detection over an extended mass range and thus the use of native conditions for sample preparation, separation (size exclusion chromatography, SEC), and ionization. The unreduced data (time-domain transients) were acquired with an external high-performance data acquisition system (FTMS Booster X2, Spectroswiss) and processed using the advanced FTMS data processing and analysis software package Peak-byPeak (Spectroswiss). The ability to define the resolution of the final mass spectrum was achieved by a user-controlled time-domain transient truncation process - as FT mass spectral resolution is proportional to the transient period, typically. ${ }^{[62]}$ In the case of mAbs, the latter statement is fulfilled if short, less than $60 \mathrm{~ms}$, transient periods are considered. ${ }^{[46]}$ This period corresponds to the half-width of the first time-domain isotopic beat of a mAb. However, increasing the transient period, even substantially, by 10 -fold and more, will not enhance resolution. Again, this is due to the particular structure of the isotopic beat patterns from mAbs in FTMS.[17d,63]

In the above example, the identified optimum mass spectral resolution that enabled estimation of DAR values of the investigated AOC sample was 4-fold lower than the lowest resolution provided by the employed Orbitrap model. It is also 2-fold lower than the lowest resolution provided by any current Orbitrap model. This example illustrates the additional flexibility and intriguing potential toward developing novel methods allowed by the access and possibility to process unreduced FTMS data (time-domain transients). This is readily available nowadays, for example, via the new generation of high-performance data acquisition and data processing systems developed by Spectroswiss. ${ }^{[47]}$

Finally, we demonstrated that the accuracy with which the DAR values could be assigned for ADCs/AOCs samples depends on the data processing of the LC-MS data. Specifically, DAR results obtained from mass spectral averaging across a complete elution peak may significantly, by more than $10 \%$, differ from the results obtained with the sliding window approach. In the latter method, the whole elution profile for a given peak is divided into several, e.g., 8-10, narrower elution time windows. Mass spectral averaging is first performed within the individual elution time windows, and the DAR distribution is calculated for each of these windows. The final DAR distribution is an average of all the window-specific DAR distributions. Previously, the sliding window approach has been reported in the peer-reviewed literature for the analysis of mAbs, ${ }^{[64]}$ but not the ADCs/AOCs. As it could be expected from the native SEC-FTMS measurements, processing mass spectra from different portions of the elution profile showed that the heavier species, with DAR $=6-8$, elute first, whereas the lighter ones, with DAR $=1-3$, are detected at the end of the elu- tion peak. ${ }^{[46]}$ The species with the highest and lowest DAR values are those that substantially differ from the whole elution peak integration analysis. It appears that mass spectral averaging of the whole elution profile suppresses ion signals from the species with the lowest and the highest DARs. Therefore, the sliding window approach has demonstrated a potential increase in DAR estimation accuracy in the ADC/AOC sample analysis. Further method validation and benchmarking are thus justified.

\section{Further Considerations}

Instrumentation advances in MS techniques, combined with gas-phase ion activation and dissociation method improvements for MS/MS, and the allied developments in data processing and data analysis, as well as liquid-phase sample preparation and separation, have propelled the structural analysis of individual mAbs and other biotherapeutics to new heights in efficiency, accuracy, and robustness. Overall, these advances have facilitated the acceptance of MS-based methods to characterize mAb-based biotherapeutics both in the biopharma industry and regulatory agencies, such as the Food and Drug Administration (FDA) and European Medicines Agency (EMA). Following the success of bottom-up MS approaches that are now integrated into the multiattribute method (MAM) workflows increasingly employed by biopharma, ${ }^{[10 b, 65]}$ the intact mass measurements and the middleup/down workflows are now being considered to be integrated into the MAM-type workflows. ${ }^{[10 a]}$ Today, for many routine biotherapeutics characterization processes, the current bottlenecks are within the automation and integration of the experimental and data analysis workflows, rather than in the MS method development itself. Nevertheless, further progress in the MS-based mAb structural analysis remains desirable, particularly in the mAb development activities, but requires addressing numerous challenges in methods and techniques. ${ }^{[12 b, 66]}$

The top-down MS analysis of mAbs remains a niche application. It still cannot yield $80-100 \%$ sequence coverage even for a highly purified and unlimited amount mAb sample. Achieving this objective would require developing efficient multi-stage MS/ MS methods that employ both types of MS/MS techniques - ergodic (e.g., CID) and non-ergodic (e.g., ECD) - consecutively and in a single experimental set-up. Perhaps a combination of a multi-trap MS/MS device (Omnitrap ${ }^{\mathrm{TM}}$ from FasmaTech, Athens, Greece) with an Orbitrap platform will offer the most advanced capabilities that will help to achieve these objectives already in the near future.

The compelling capabilities offered by the use of the unreduced data in FTMS, achieved via the acquisition and rational processing of the time-domain transients, have already resulted in the development of novel methods, such as the proteoform peak integration approach for ADC/AOC analysis of DARs. ${ }^{[46]}$ Furthermore, access to the time-domain transients enables the implementation and use of such intriguing novel methods as individual ion counting (I2MS), ${ }^{[67]}$ and charge determination analysis (CHARDA). ${ }^{68]}$ These methods address one of the major bottlenecks in top-down MS by facilitating analysis of the heavily congested mass spectra. In the I2MS approach, peaks of individual multiply-charged ions are detected in the $\mathrm{m} / \mathrm{z}$ space. The charge states are assigned to these peaks based on the growth rate of the peak intensity as a function of the transient period. This rate is proportional to the charge state - peaks with higher charge states increase their abundances faster. The approach relies on statistics and requires that the individual ions survive during extended detection periods. The second approach, CHARDA, processes timedomain transients from an inverse perspective - it monitors the decay rates of ion signals along the time-domain transients. Ions with different charge states have been shown to have different decay rates. The benefit of CHARDA is that individual ion detection is not required, and a top-down MS experiment can be conducted 
conventionally. Therefore, CHARDA can assign charge states to the stand-alone peaks composed of many (and not necessarily high charge state) ions, which may enhance method sensitivity and widen charge state applicability.

Furthermore, a robust and rapid top-down de novo sequencing of IgGs is not yet offered for the biologically-significant cases, especially when the antibody quantity is limited and efficient purification or production of an IgG of interest is not possible. To achieve this objective, exceptional mass accuracy is needed to be provided for both canonical (i.e., $\mathrm{N}$ - and $\mathrm{C}$-terminal) and internal product ions in the top-down data sets. ${ }^{[69]}$ According to the current estimates, mass accuracy of better than $1 \mathrm{ppm}$ is indispensable for increasing confidence, specifically in product ion annotation. ${ }^{[70]}$ That signifies a 5-10-fold improvement over the current stateof-the-art. With the high resolution and high mass accuracy data in hand, sophisticated top-down de novo sequencing algorithms, such as Twister, ${ }^{[71]}$ may receive the much-needed performance enhancement. In addition, increased sequencing confidence and, potentially, facilitated de novo sequencing, can be accomplished by reducing congestion of product ions upon distributing them across a wider $m / z$ range. ${ }^{[72]}$

Finally, achieving the top-down MS capabilities overviewed here at the level of mAb mixtures is still beyond the reach of the current analytical technology. Nevertheless, recent developments have already demonstrated proof-of-concept capabilities of a middle-down analysis (on the level of $\mathrm{F}(\mathrm{ab})$ subunits) to probe the circulating IgG1 repertoire in human plasma. ${ }^{[73]} \mathrm{In}$ addition to the MS and MS/MS challenges, the liquid-phase separation of IgGs presents a formidable bottleneck due to the similar physico-chemical properties of IgGs, including their pI values. Implementation of efficient methods to separate complex mixtures of mAbs in solution and/or in the gas phase (e.g., using ion mobility $)^{[74]}$ will trigger a significant advancement of the already developed MS and MS/MS-based workflows for the analysis of IgGs in general, and $\mathrm{mAbs}$ in particular, embedded in complex molecular matrices and accelerate our access to new medicines.

\section{Conclusions}

The growing importance of mAbs/ADCs as biotherapeutics has magnified the usefulness of top-down and middle-down mass spectrometry for their structural analysis, including those methods developed by our group (Table 1). Notably, we pioneered topdown and IdeS/KGP-based middle-down MS approaches to mAb analysis utilizing ETD as a gas-phase ion fragmentation method on two platforms: TOF MS and Orbitraps. The general character of the allied approaches to increase the sensitivity and specificity of $\mathrm{mAb} / \mathrm{ADC}$ analysis, including data averaging across multiple technical replicates and the use of unreduced data in FTMS, further expands the application reach of these methods (Table 1). The ideas behind several developed methods and techniques have been employed in related commercial products and are readily available to the community.

Nevertheless, a global and concerted effort is needed to advance the field of top-down MS toward routine workflows with a broad acceptance by the larger scientific, industrial, and regulatory communities. We contribute to this process by participating in the Consortium for Top-Down Proteomics (CTDP) and other initiatives focused on further developing and promoting top-down MS approaches. In the frame of the CTDP activities, we supervised an interlaboratory study on the characterization of mAbs with top-down and middle-down MS. ${ }^{[12 b]}$ This study summarized $\mathrm{mAb}$ analysis reports from more than 20 top-down MS laboratories worldwide. It underlined a particular need in didactic and educational resources, supported with software tools, to more rigorously comprehend the structural chemistry and mass spectra of large molecules and improve the experimental design. To address this, we developed a dedicated software tool, FTMS Simulator, capable of accurately computing mAb's isotopic envelopes and simulating FTMS-specific data for biotherapeutics analysis. ${ }^{[46,75]}$

Presently, we are assisting the major CTDP action in advancing the Human Proteoform Project - an ambitious initiative to bridge genotype and phenotype via large-scale proteome analysis at the proteoform level with top-down MS. ${ }^{[76]}$ Advances in the latter will be necessarily beneficial for the whole field of top-down MS, including large-scale and targeted biopharma applications.

\section{Acknowledgments}

We dedicate this article to the memory of Prof. Fred W. McLafferty to commemorate his outstanding contributions and achievements in the field of top-down mass spectrometry. We heartily thank Dr. Unige Laskay and the other team members of our $\mathrm{mAb}$ research program at Biomolecular Mass Spectrometry Laboratory at Ecole Polytechnique Fédérale de Lausanne (EPFL). We greatly appreciate our collaboration for Sap9 production and characterization with Dr. Michel Monod from CHUV, Lausanne. We also express our gratitude to Bruker Daltonics and Thermo Fisher Scientific for fruitful collaboration and support. We thank Spectroswiss, EPFL, the Swiss National Science Foundation (grant \#147006 to YOT), and the European Horizon 2020 research and innovation program's projects \#829157 (TopSpec) and \#964553 (ARIADNE) for funding.

Received: November 14, 2021

[1] H. Kaplon, J. M. Reichert, mAbs 2021, 13, 1860476 , https://doi.org/10.1080/19420862.2020.1860476.

[2] L. M. Smith, N. L. Kelleher, P. The Consortium for Top Down, M. Linial, D. Goodlett, P. Langridge-Smith, Y. Ah Goo, G. Safford, L. Bonilla, G. Kruppa, R. Zubarev, J. Rontree, J. Chamot-Rooke, J. Garavelli, A. Heck, J. Loo, D. Penque, M. Hornshaw, C. Hendrickson, L. Paša-Tolić, C. Borchers, D. Chan, N. Young, J. Agar, C. Masselon, M. Gross, F. McLafferty, Y. Tsybin, Y. Ge, I. Sanders, J. Langridge, J. Whitelegge, A. Marshall, Nat. Methods 2013, 10, 186, https://doi.org/10.1038/nmeth.2369.

[3] Z. Zhang, H. Pan, X. Chen, Mass Spectrom. Rev. 2009, 28, 147, https://doi.org/10.1002/mas.20190.

[4] R. Plomp, G. Dekkers, Y. Rombouts, R. Visser, C. A. M. Koeleman, G. S. M. Kammeijer, B. C. Jansen, T. Rispens, P. J. Hensbergen, G. Vidarsson, M. Wuhrer, Mol. Cell. Proteomics 2015, 14, 1373, https://doi.org/10.1074/mcp.M114.047381.

[5] G. Vidarsson, G. Dekkers, T. Rispens, Front. Immunol. 2014, 5, https://doi.org/10.3389/fimmu.2014.00520.

[6] A. L. Grilo, A. Mantalaris, Trends Biotechnol. 2019, 37, 9, https://doi.org/10.1016/j.tibtech.2018.05.014.

[7] C. Gstöttner, D. Reusch, M. Haberger, I. Dragan, P. Van Veelen, D. P. A Kilgour, Y. O. Tsybin, Y. E. M. van der Burgt, M. Wuhrer, S. Nicolardi, $m A b s$ 2020, 12, 1682403, https://doi.org/10.1080/19420862.2019.1682403.

[8] a) A. Beck, L. Goetsch, C. Dumontet, N. Corvaïa, Nat. Rev. Drug Discov. 2017, 16, 315, https://doi.org/10.1038/nrd.2016.268; b) A. Wakankar, Y. Chen, Y. Gokarn, F. S. Jacobson, $m A$ s s 2011, 3, 161, https://doi.org/10.4161/mabs.3.2.14960; c) A. Beck, V. D'Atri, A Ehkirch, S. Fekete, O. Hernandez-Alba, R. Gahoual, E. Leize-Wagner, Y. François, D. Guillarme, S. Cianférani, Exp. Rev. Proteomics 2019, 16, 337, https://doi.org/10.1080/14789450.2019.1578215; d) J. DugalTessier, S. Thirumalairajan, N. Jain, J. Clin. Med. 2021, 10, 838, https://doi.org/10.3390/jcm10040838; e) I. Dovgan, O. Koniev, S. Kolodych, A. Wagner, Bioconj. Chem. 2019, 30, 2483, https://doi.org/10.1021/acs.bioconjchem.9b00306.

[9] a) M. Källsten, M. Pijnappel, R. Hartmann, F. Lehmann, L. Kovac, S. B. Lind, J. Bergquist, Anal. Bioanal. Chem. 2019, 411, 2569, https://doi.org/10.1007/s00216-019-01699-0; b) K. Xu, L. Liu, R. Dere, E. Mai, R. Erickson, A. Hendricks, K. Lin, J. R. Junutula, S. Kaur, Bioanalysis 2013, 5, 1057, https://doi.org/10.4155/bio.13.66; c) F. Debaene, A. Bœuf, E. Wagner-Rousset, O. Colas, D. Ayoub, N. Corvaïa, A. Van Dorsselaer, A. Beck, S. Cianférani, Anal. Chem. 2014, 86, 10674, https://doi.org/10.1021/ac502593n.

[10] a) J. R. Auclair, A. S. Rathore, LC-GC North America 2021, 39, 548; b) J. Auclair, A. Rathore, LC-GC North America 2021, 39, 28.

[11] Y. Xu, D. Wang, B. Mason, T. Rossomando, N. Li, D. Liu, J. K. Cheung, W. Xu, S. Raghava, A. Katiyar, C. Nowak, T. Xiang, D. D. Dong, J. Sun, A. Beck, H. Liu, mAbs 2019, 11, 239, https://doi.org/10.1080/19420862.2018.1553476.

[12] a) Y. Jin, Z. Lin, Q. Xu, C. Fu, Z. Zhang, Q. Zhang, W. A. Pritts, Y. Ge, $m A$ s 2019, 11, 106, https://doi.org/10.1080/19420862.2018.1525253; b) K. Srzentić, L. Fornelli, Y. O. Tsybin, J. A. Loo, H. Seckler, J. N. Agar, L. C. Anderson, D. L. Bai, A. Beck, J. S. Brodbelt, Y. E. M. van der Burgt, J. Chamot-Rooke, S. Chatterjee, Y. Chen, D. J. Clarke, P. O. Danis, J. K. Diedrich, R. A. D’Ippolito, M. Dupré, N. Gasilova, Y. Ge, Y. A. Goo, D. R. 
Goodlett, S. Greer, K. F. Haselmann, L. He, C. L. Hendrickson, J. D. Hinkle, M. V. Holt, S. Hughes, D. F. Hunt, N. L. Kelleher, A. N. Kozhinov, Z. Lin, C. Malosse, A. G. Marshall, L. Menin, R. J. Millikin, K. O. Nagornov, S. Nicolardi, L. Paša-Tolić, S. Pengelley, N. R. Quebbemann, A. Resemann, W. Sandoval, R. Sarin, N. D. Schmitt, J. Shabanowitz, J. B. Shaw, M. R. Shortreed, L. M. Smith, F. Sobott, D. Suckau, T. Toby, C. R. Weisbrod, N. C. Wildburger, J. R. Yates, S. H. Yoon, N. L. Young, M. Zhou, J. Am. Soc. Mass Spectrom. 2020, 31, 1783, https://doi.org/10.1021/jasms.0c00036; c) M. L. A. De Leoz, D. L. Duewer, S. E. Stein, NISTIR 8186 2017, https://doi.org/10.6028/NIST.IR.8186; d) T. Mouchahoir, J. E. Schiel, R. Rogers, A. Heckert, B. J. Place, A. Ammerman, X. Li, T. Robinson, B. Schmidt, C. M. Chumsae, X. Li, A. V. Manuilov, B. Yan, G. O. Staples, D. Ren, A. J. Veach, D. Wang, W. Yared, Z. Sosic, Y. Wang, L. Zang, A. M. Leone, P. Liu, R. Ludwig, L. Tao, W. Wu, A. Cansizoglu, A. Hanneman, G. W. Adams, I. Perdivara, H. Walker, M. Wilson, A. Brandenburg, N. DeGraanWeber, S. Gotta, J. Shambaugh, M. Alvarez, X. C. Yu, L. Cao, C. Shao, A. Mahan, H. Nanda, K. Nields, N. Nightlinger, H. M. Barysz, M. Jahn, B. Niu, J. Wang, G. Leo, N. Sepe, Y.-H. Liu, B. A. Patel, D. Richardson, Y. Wang, D. Tizabi, O. V. Borisov, Y. Lu, E. L. Maynard, A. Gruhler, K. F. Haselmann, T. N. Krogh, C. P. Sönksen, S. Letarte, S. Shen, K. Boggio, K. Johnson, W. Ni, H. Patel, D. Ripley, J. C. Rouse, Y. Zhang, C. Daniels, A. Dawdy, O. Friese, T. W. Powers, J. B. Sperry, J. Woods, E. Carlson, K. I. Sen, S. J. Skilton, M. Busch, A. Lund, M. Stapels, X. Guo, S. Heidelberger, H. Kaluarachchi, S. McCarthy, J. Kim, J. Zhen, Y. Zhou, S. Rogstad, X. Wang, J. Fang, W. Chen, Y. Q. Yu, J. G. Hoogerheide, R. Scott, H. Yuan, J. Am. Soc. Mass Spectrom. 2021, 32, 913, https://doi.org/10.1021/jasms.0c00415.

[13] a) B. Bobály, S. Fleury-Souverain, A. Beck, J.-L. Veuthey, D. Guillarme, S. Fekete, J. Pharmaceut. Biomed. Anal. 2018, 147, 493, https://doi.org/10.1016/j.jpba.2017.06.022; b) Y. Matsuda, V. Robles, M.C. Malinao, J. Song, B. A. Mendelsohn, Anal. Chem. 2019, 91, 12724, https://doi.org/10.1021/acs.analchem.9b02192; c) R. S. Rogers, N. S. Nightlinger, B. Livingston, P. Campbell, R. Bailey, A. Balland, $m A b s$ 2015, 7, 881, https://doi.org/10.1080/19420862.2015.1069454.

[14] X. Li, F. Wang, W. Xu, K. May, D. Richardson, H. Liu, Anal. Biochem. 2013, 436, 93, https://doi.org/10.1016/j.ab.2013.01.020.

[15] a) Y. Leblanc, C. Ramon, N. Bihoreau, G. Chevreux, J. Chromatogr. B 2017, 1048, 130, https://doi.org/10.1016/j.jchromb.2017.02.017; b) R. Y. C. Huang, E. G. Deyanova, D. Passmore, V. Rangan, S. Deshpande, A. A. Tymiak, G. Chen, J. Am. Soc. Mass Spectrom. 2015, 26, 1791, https://doi.org/10.1021/jasms.8b04893; c) L. A. Khawli, S. Goswami, R. Hutchinson, Z. W. Kwong, J. Yang, X. Wang, Z. Yao, A. Sreedhara, T. Cano, D. B. Tesar, I. Nijem, D. E. Allison, P. Y. Wong, Y.-H. Kao, C. Quan, A. Joshi, R. J. Harris, P. Motchnik, mAbs 2010, 2, 613 , https://doi.org/10.4161/mabs.2.6.13333; d) L. Tang, S. Sundaram, J. Zhang, P. Carlson, A. Matathia, B. Parekh, Q. Zhou, M.-C. Hsieh, mAbs 2013, 5, 114, https://doi.org/10.4161/mabs.22695.

[16] A. Beck, G. Terral, F. Debaene, E. Wagner-Rousset, J. Marcoux, M.-C. Janin-Bussat, O. Colas, A. V. Dorsselaer, S. Cianférani, Exp. Rev. Proteomics 2016, 13, 157, https://doi.org/10.1586/14789450.2016.1132167.

[17] a) M. Scigelova, M. Hornshaw, A. Giannakopulos, A. Makarov, Mol. Cell. Proteomics 2011, 10, M111.009431, https://doi.org/10.1074/mcp.M111.009431; b) R. A. Zubarev, A. Makarov, Anal. Chem. 2013, 85, 5288, https://doi.org/10.1021/ ac4001223; c) A. Makarov, E. Denisov, O. Lange, J. Am. Soc. Mass Spectrom. 2009, 20, 1391, https://doi.org/10.1016/j.jasms.2009.01.005; d) A. Makarov, E. Denisov, J. Am. Soc. Mass Spectrom. 2009, 20, 1486, https://doi.org/10.1016/j.jasms.2009.03.024; e) F. Xian, C. L. Hendrickson, A. G. Marshall, Anal. Chem. 2012, 84, 708, https://doi.org/10.1021/ac203191t; f) C. L. Hendrickson, J. P. Quinn, N. K. Kaiser, D. F. Smith, G. T. Blakney, T. Chen, A. G. Marshall, C. R. Weisbrod, S. C. Beu, J. Am. Soc. Mass Spectrom. 2015, 26, 1626, https://doi.org/10.1007/s13361-015-1182-2; g) L. Fornelli, J. Parra, R. Hartmer, C. Stoermer, M. Lubeck, Y. O. Tsybin, Anal. Bioanal. Chem. 2013, 405, 8505, https://doi.org/10.1007/s00216-013-72675; h) T. Baba, P. Ryumin, E. Duchoslav, K. Chen, A. Chelur, B. Loyd, I. Chernushevich, J. Am. Soc. Mass Spectrom. 2021, 32, 1964 , https://doi.org/10.1021/jasms.0c00425.

[18] a) I. D. G. Campuzano, W. Sandoval, J. Am. Soc. Mass Spectrom. 2021, https://doi.org/10.1021/jasms.1c00036; b) A. C. Leney, A. J. R. Heck, J. Am. Soc. Mass Spectrom. 2017, 28, 5, https://doi.org/10.1007/s13361-016-1545-3; c) S. Tamara, M. A. den Boer, A. J. R. Heck, Chem. Rev. 2021, https://doi.org/10.1021/acs.chemrev.1c00212.

[19] K. O. Zhurov, L. Fornelli, M. D. Wodrich, U. A. Laskay, Y. O. Tsybin, Chem. Soc. Rev. 2013, 42, 5014, https://doi.org/10.1039/C3CS35477F.

[20] a) Y. O. Tsybin, P. Håkansson, B. A. Budnik, K. F. Haselmann, F. Kjeldsen, M. Gorshkov, R. A. Zubarev, Rapid Commun. Mass Spectrom. 2001, 15, 1849, https://doi.org/10.1002/rcm.448; b) J. E. P. Syka, J. J. Coon, M. J. Schroeder, J. Shabanowitz, D. F. Hunt, Proc. Natl. Acad. Sci. USA 2004, 101, 9528, https://doi.org/10.1073/pnas.0402700101; c) R. A. Zubarev, A. R. Zubarev, M. M. Savitski, J. Am. Soc. Mass Spectrom. 2008, 19, 753, https://doi.org/10.1016/j.jasms.2008.03.007; d) N. M. Riley, M. S. Westphall, J. J. Coon, Anal. Chem. 2015, 87, 7109, https://doi.org/10.1021/acs.analchem.5b00881; e) H. J. Cooper, K. Hakansson, A. G. Marshall, Mass Spectrom. Rev. 2005, 24, 201.

[21] a) S. Nicolardi, L. Switzar, A. M. Deelder, M. Palmblad, Y. E. M. van der Burgt, Anal. Chem. 2015, 87, 3429, https://doi.org/10.1021/ac504708y; b) Y. E. M. van der Burgt, D. P. A. Kilgour, Y. O. Tsybin, K. Srzentić, L. Fornelli, A. Beck, M. Wuhrer, S. Nicolardi, Anal. Chem. 2019, 91, 2079, https://doi.org/10.1021/acs.analchem.8b04515.

[22] a) T. P. Cleland, C. J. DeHart, R. T. Fellers, A. J. VanNispen, J. B. Greer, R. D. LeDuc, W. R. Parker, P. M. Thomas, N. L. Kelleher, J. S. Brodbelt, J. Proteome Res. 2017, 16, 2072, https://doi.org/10.1021/acs.jproteome.7b00043; b) L. Fornelli, K. Srzentić, R. Huguet, C. Mullen, S. Sharma, V. Zabrouskov, R. T. Fellers, K. R. Durbin, P. D. Compton, N. L. Kelleher, Anal. Chem. 2018, 90, 8421, https://doi.org/10.1021/acs.analchem.8b00984.

[23] A. Q. Stiving, Z. L. VanAernum, F. Busch, S. R. Harvey, S. H. Sarni, V. H. Wysocki, Anal. Chem. 2019, 91, 190, https://doi.org/10.1021/acs.analchem.8b05071.

[24] R. Huguet, C. Mullen, K. Srzentić, J. B. Greer, R. T. Fellers, V. Zabrouskov, J. E. P. Syka, N. L. Kelleher, L. Fornelli, Anal. Chem. 2019, 91, 15732, https://doi.org/10.1021/acs.analchem.9b03925.

[25] J. Sjögren, L. Andersson, M. Mejàre, F. Olsson, in 'Bacterial Pathogenesis: Methods and Protocols', Eds. P. Nordenfelt, M. Collin, Springer New York, New York, NY, 2017, p. 319, https://doi.org/10.1007/978-1-4939-6673-8_21.

[26] F. Lermyte, Y. O. Tsybin, P. B. O'Connor, J. A. Loo, J. Am. Soc. Mass Spectrom. 2019, https://doi.org/10.1007/s13361-019-02201-x.

[27] L. M. Smith, P. M. Thomas, M. R. Shortreed, L. V. Schaffer, R. T. Fellers, R. D. LeDuc, T. Tucholski, Y. Ge, J. N. Agar, L. C. Anderson, J. ChamotRooke, J. Gault, J. A. Loo, L. Paša-Tolić, C. V. Robinson, H. Schlüter, Y. O. Tsybin, M. Vilaseca, J. A. Vizcaíno, P. O. Danis, N. L. Kelleher, Nat. Methods 2019, 16, 939, https://doi.org/10.1038/s41592-019-0573-x.

[28] P. V. Bondarenko, T. P. Second, V. Zabrouskov, A. A. Makarov, Z. Zhang, J. Am. Soc. Mass Spectrom. 2009, 20, 1415 , https://doi.org/10.1016/j.jasms.2009.03.020.

[29] Y. O. Tsybin, L. Fornelli, C. Stoermer, M. Luebeck, J. Parra, S. Nallet, F. M. Wurm, R. Hartmer, Anal. Chem. 2011, 83, 8919, https://doi.org/10.1021/ac201293m.

[30] X. Han, M. Jin, K. Breuker, F. W. McLafferty, Science 2006, 314, 109, https://doi.org/10.1126/science.1128868.

[31] Y. Ge, I. N. Rybakova, Q. Xu, R. L. Moss, Proc. Natl. Acad. Sci. USA 2009, 106, 12658, https://doi.org/10.1073/pnas.0813369106.

[32] J. S. Beckman, V. G. Voinov, M. Hare, D. Sturgeon, Y. Vasil'ev, D. Oppenheimer, J. B. Shaw, S. Wu, R. Glaskin, C. Klein, C. Schwarzer, G. Stafford, J. Am. Soc. Mass Spectrom. 2021, 32, 2081, https://doi.org/10.1021/jasms.0c00482.

[33] a) Y. O. Tsybin, K. F. Haselmann, M. R. Emmett, C. L. Hendrickson, A. G. Marshall, J. Am. Soc. Mass Spectrom. 2006, 17, 1704, https://doi.org/10.1016/j.jasms.2006.07.024; b) A. Vorobyev, H. B Hamidane, Y. O. Tsybin, J. Am. Soc. Mass Spectrom. 2009, 20, 2273, https://doi.org/10.1016/j.jasms.2009.08.019.

[34] M. A. den Boer, J.-F. Greisch, S. Tamara, A. Bondt, A. J. R. Heck, Chem. Sci. 2020, 11, 11886, https://doi.org/10.1039/D0SC03438J.

[35] L. Fornelli, E. Damoc, P. M. Thomas, N. L. Kelleher, K. Aizikov, E. Denisov, A. Makarov, Y. O. Tsybin, Mol. Cell. Proteomics 2012, 11, 1758, https://doi.org/10.1074/mcp.M112.019620.

[36] Y. Qi, P. B. O'Connor, Mass Spectrom. Rev. 2014, 33, 333, https://doi.org/10.1002/mas.21414.

[37] Y. O. Tsybin, K. O. Nagornov, A. N. Kozhinov, in 'Fundamentals and Applications of Fourier Transform Mass Spectrometry', Eds. B. Kanawati, P. Schmitt-Kopplin, Elsevier, 2019, p. 113, https://doi.org/10.1016/B978-0-12-814013-0.00005-3.

[38] Y. Mao, S. G. Valeja, J. C. Rouse, C. L. Hendrickson, A. G. Marshall, Anal. Chem. 2013, 85, 4239, https://doi.org/10.1021/ac303525n.

[39] O. Lange, E. Damoc, A. Wieghaus, A. Makarov, Int. J. Mass Spectrom. 2014, 369, 16, https://doi.org/10.1016/j.ijms.2014.05.019.

[40] a) K. O. Zhurov, A. N. Kozhinov, Y. O. Tsybin, Energy \& Fuels 2013 27, 2974; b) K. Schuhmann, K. Srzentić, K. O. Nagornov, H. Thomas, T. Gutmann, Ü. Coskun, Y. O. Tsybin, A. Shevchenko, Anal. Chem. 2017, 89, 12857, https://doi.org/10.1021/acs.analchem.7b03437.

[41] L. Fornelli, D. Ayoub, K. Aizikov, X. Liu, E. Damoc, P. A. Pevzner, A. Makarov, A. Beck, Y. O. Tsybin, J. Proteomics 2017, 159, 67, https://doi.org/10.1016/j.jprot.2017.02.013.

[42] K. Scheffler, R. Viner, E. Damoc, J. Proteomics 2018, 175, 42, https://doi.org/10.1016/j.jprot.2017.03.028.

[43] B. Wang, A. C. Gucinski, D. A. Keire, L. F. Buhse, M. T. Boyne Ii, Analyst 2013, 138, 3058, https://doi.org/10.1039/C3AN36524G.

[44] L. Fornelli, D. Ayoub, K. Aizikov, A. Beck, Y. O. Tsybin, Anal. Chem. 2014, 86, 3005, https://doi.org/10.1021/ac4036857.

[45] K. Srzentić, K. O. Nagornov, L. Fornelli, A. A. Lobas, D. Ayoub, A. N. Kozhinov, N. Gasilova, L. Menin, A. Beck, M. V. 
Gorshkov, K. Aizikov, Y. O. Tsybin, Anal. Chem. 2018, 90, 12527, https://doi.org/10.1021/acs.analchem.8b02398.

[46] K. O. Nagornov, N. Gasilova, A. N. Kozhinov, P. Virta, P Holm, L. Menin, V. J. Nesatyy, Y. O. Tsybin, Anal. Chem. 2021, https://doi.org/10.1021/acs.analchem.1c02247.

[47] a) J. R. Bills, K. O. Nagornov, A. N. Kozhinov, T. J. Williams, Y. O. Tsybin, R. K. Marcus, J. Am. Soc. Mass Spectrom. 2021, https://doi.org/10.1021/jasms.1c00051; b) K. O. Nagornov, M. Zennegg, A. N. Kozhinov, Y. O. Tsybin, D. Bleiner, J. Am. Soc. Mass Spectrom. 2020, 31 , 257, https://doi.org/10.1021/jasms.9b00032.

[48] a) J. Kim, L. Jones, L. Taylor, G. Kannan, F. Jackson, H. Lau, R. F. Latypov, B. Bailey, J. Chromatogr. B 2010, 878, 1973, https://doi.org/10.1016/j.jchromb.2010.05.032; $\quad$ b) $\quad$ G. $\quad$ D. Pipes, P. Campbell, P. V. Bondarenko, B. A. Kerwin, M. J. Treuheit, H. S. Gadgil, J. Pharmaceut. Sci. 2010, 99, 4469, https://doi.org/10.1002/jps.22158; c) F. Rinaldi, S. Tengattini, G. Brusotti, G. Tripodo, B. Peters, C. Temporini, G. Massolini, E. Calleri, Front. Mol. Biosci. 2021, 8, https://doi.org/10.3389/fmolb.2021.765683.

[49] J. J. Cournoyer, J. L. Pittman, V. B. Ivleva, E. Fallows, L. Waskell, C. E. Costello, P. B. O'Connor, Protein Sci. 2005, 14, 452, https://doi.org/10.1110/ps.041062905.

[50] U. Laskay, A., K. Srzentic, L. Fornelli, O. Upir, A. N. Kozhinov, M. Monod, Y. O. Tsybin, CHIMIA 2013, 67, 244, https://doi.org/10.2533/chimia.2013.244.

[51] Ü. A. Laskay, K. Srzentić, M. Monod, Y. O. Tsybin, J. Proteomics 2014, 110, 20, https://doi.org/10.1016/j.jprot.2014.07.035.

[52] K. Srzentić, L. Fornelli, Ü. A. Laskay, M. Monod, A. Beck, D. Ayoub, Y. O. Tsybin, Anal. Chem. 2014, 86, 9945, https://doi.org/10.1021/ac502766n.

[53] K. Vyatkina, L. J. M. Dekker, S. Wu, M. M. VanDuijn, X. Liu, N. Tolić, T. M. Luider, L. Paša-Tolić, Proteomics 2017, 17, 1600321, https://doi.org/10.1002/pmic.201600321.

[54] K. I. Sen, W. H. Tang, S. Nayak, Y. J. Kil, M. Bern, B. Ozoglu, B. Ueberheide, D. Davis, C. Becker, J. Am. Soc. Mass Spectrom. 2017, 28, 803, https://doi.org/10.1007/s13361-016-1580-0.

[55] L. Kang, N. Weng, W. Jian, Biomed. Chromatogr. 2020, 34, e4633, https://doi.org/10.1002/bmc.4633.

[56] P. Bults, B. Spanov, O. Olaleye, N. C. van de Merbel, R. Bischoff, J. Chromatogr. B 2019, 1110-1111, 155, https://doi.org/10.1016/j.jchromb.2019.01.032.

[57] S. Nallet, L. Fornelli, S. Schmitt, J. Parra, L. Baldi, Y. O. Tsybin, F. M. Wurm, New Biotechnol. 2012, 29, 471, https://doi.org/10.1016/j.nbt.2012.02.003.

[58] a) L. Fornelli, K. R. Durbin, R. T. Fellers, B. P. Early, J. B. Greer, R. D. LeDuc, P. D. Compton, N. L. Kelleher, J. Proteome Res. 2017, 16, 609, https://doi.org/10.1021/acs.jproteome.6b00698; b) P. D. Compton, L. Zamdborg, P. M. Thomas, N. L. Kelleher, Anal. Chem. 2011, 83, 6868, https://doi.org/10.1021/ac2010795.

[59] a) A. Beck, H. Liu, Antibodies (Basel) 2019, 8, 18, https://doi.org/10.3390/antib8010018; b) V. Faid, Y. Leblanc, N. Bihoreau, G. Chevreux, J. Pharmaceut. Biomed. Anal. 2018, 149, 541, https://doi.org/10.1016/j.jpba.2017.11.046.

[60] a) A. Resemann, W. Jabs, A. Wiechmann, E. Wagner, O. Colas, W. Evers, E. Belau, L. Vorwerg, C. Evans, A. Beck, D. Suckau, mAbs 2016, $8, \quad 318, \quad$ https://doi.org/10.1080/19420862.2015.1128607; b) D. Ayoub, W. Jabs, A. Resemann, W. Evers, C. Evans, L. Main, C. Baessmann, E. Wagner-Rousset, D. Suckau, A. Beck, $m A$ bs 2013, 5, 699, https://doi.org/10.4161/mabs.25423.

[61] a) A. D. Rolland, J. S. Prell, Chem. Rev. 2021, https://doi.org/10.1021/acs.chemrev.1c00696; b) I. D. G. Campuzano, C. Netirojjanakul, M. Nshanian, J. L. Lippens, D. P. A. Kilgour, S. Van Orden, J. A. Loo, Anal. Chem. 2018, 90, 745, https://doi.org/10.1021/acs.analchem.7b03021.

[62] A.G.Marshall,C.L.Hendrickson,G.S.Jackson,MassSpectrom.Rev.1998, 17,1, https://doi.org/10.1002/(sici)1098-2787(1998)17:1<1::aid-mas1>3.0.co;2-k.

[63] a) S. A. Hofstadler, J. E. Bruce, A. L. Rockwood, G. A. Anderson, B. E. Winger, R. D. Smith, Int. J. Mass Spectrom. Ion Proc. 1994, 132, 109, https://doi.org/10.1016/0168-1176(94)85020-8; b) M. L. Easterling, I. J. Amster, G. J. van Rooij, R. M. A. Heeren, J. Am. Soc. Mass Spectrom. 1999, 10, 1074, https://doi.org/10.1016/S1044-0305(99)00091-4.

[64] S. Millán-Martín, S. Carillo, F. Füssl, J. Sutton, P. Gazis, K. Cook, K. Scheffler, J. Bones, Eur. J. Pharmaceut. Biopharmaceut. 2021, 158, 83, https://doi.org/10.1016/j.ejpb.2020.11.006.
[65] R. S. Rogers, M. Abernathy, D. D. Richardson, J. C. Rouse, J. B. Sperry, P. Swann, J. Wypych, C. Yu, L. Zang, R. Deshpande, AAPS J. 2017, 20, 7 , https://doi.org/10.1208/s12248-017-0168-3.

[66] D. P. Donnelly, C. M. Rawlins, C. J. DeHart, L. Fornelli, L. F. Schachner, Z. Lin, J. L. Lippens, K. C. Aluri, R. Sarin, B. Chen, C. Lantz, W. Jung, K. R. Johnson, A. Koller, J. J. Wolff, I. D. G. Campuzano, J. R. Auclair, A. R. Ivanov, J. P. Whitelegge, L. Paša-Tolić, J. Chamot-Rooke, P. O. Danis, L. M. Smith, Y. O. Tsybin, J. A. Loo, Y. Ge, N. L. Kelleher, J. N. Agar, Nat. Methods 2019, 16, 587, https://doi.org/10.1038/s41592-019-0457-0.

[67] a) J. O. Kafader, R. D. Melani, K. R. Durbin, B. Ikwuagwu, B. P. Early, R. T. Fellers, S. C. Beu, V. Zabrouskov, A. A. Makarov, J. T. Maze, D. L. Shinholt, P. F. Yip, D. Tullman-Ercek, M. W. Senko, P. D. Compton, N. L. Kelleher, Nat. Methods 2020, 17, 391, https://doi.org/10.1038/s41592-020-0764-5; b) S.-H. Lai, S. Tamara, A. J. R. Heck, iScience 2021, 24, https://doi.org/10.1016/j.isci.2021.103211.

[68] Y. Lyutvinskiy, K. O. Nagornov, A. N. Kozhinov, N. Gasilova, L. Menin, Z. Meng, X. Zhang, A. Ata Saei, Y. O. Tsybin, A. Makarov, R. A. Zubarev, ChemRxiv. 2021

[69] M. A. Zenaidee, B. Wie, C. Lantz, H. T. Wu, T. R. Lambeth, J. K. Diedrich, R. R. Ogorzalek Loo, R. R. Julian, J. A. Loo, J. Am. Soc. Mass Spectrom. 2021, 32, 1752, https://doi.org/10.1021/jasms.1c00113.

[70] L. He, C. R. Weisbrod, A. G. Marshall, Int. J. Mass Spectrom. 2018, 427, 107, https://doi.org/10.1016/j.ijms.2017.11.012

[71] a) K. Vyatkina, S. Wu, L. J. M. Dekker, M. M. VanDuijn, X. Liu, N. Toli?, T. M. Luider, L. Paša-Tolić, P. A. Pevzner, Bioinformatics 2016, 32, 2753, https://doi.org/10.1093/bioinformatics/btw307; b) K. Vyatkina, Proteomes 2017, 5, 6 .

[72] J. T. Kline, C. Mullen, K. R. Durbin, R. N. Oates, R. Huguet, J. E. P. Syka, L. Fornelli, J. Am. Soc. Mass Spectrom. 2021, 32, 2334, https://doi.org/10.1021/jasms.1c00062.

[73] a) A. Bondt, M. Hoek, S. Tamara, B. de Graaf, W. Peng, D. Schulte, D. M. H. van Rijswijck, M. A. den Boer, J.-F. Greisch, M. R. J. Varkila, J. Snijder, O. L. Cremer, M. J. M. Bonten, A. J. R. Heck, Cell Sys. 2021, https://doi.org/10.1016/j.cels.2021.08.008; b) R. D. Melani, B. J. Des Soye, J. O. Kafader, E. Forte, M. Hollas, V. Blagojevic, F. Negrão, J. P. McGee, B. Drown, C. Lloyd-Jones, H. S. Seckler, J. M. Camarillo, P. D. Compton, R. D. LeDuc, B. Early, R. T. Fellers, B.-K. Cho, B. B Mattamana, Y. A. Goo, P. M. Thomas, M. K. Ash, P. P. Bhimalli, L. AlHarthi, B. E. Sha, J. R. Schneider, N. L. Kelleher, J. Proteome Res. 2021 https://doi.org/10.1021/acs.jproteome.1c00882.

[74] a) P. Pathak, A. A. Shvartsburg, Anal. Chem. 2020, 92, 13855 , https://doi.org/10.1021/acs.analchem.0c02551; b) R. D. Melani, K. Srzentić, V. R. Gerbasi, J. P. McGee, R. Huguet, L. Fornelli, N. L. Kelleher, $m A b s$ 2019, 11, 1351, https://doi.org/10.1080/19420862.2019.1668226.

[75] K. O. Nagornov, A. N. Kozhinov, N. Gasilova, L. Menin, Y. O. Tsybin, J. Am. Soc. Mass Spectrom. 2020, 31, 1927, https://doi.org/10.1021/jasms.0c00190.

[76] L. M. Smith, J. N. Agar, J. Chamot-Rooke, P. O. Danis, Y. Ge, J. A. Loo, L. Paša-Tolić, Y. O. Tsybin, N. L. Kelleher, Sci. Adv. 2021, 7, 1, https://doi.org/doi:10.1126/sciadv.abk0734.

[77] R. Feng, Y. Konishi, Anal. Chem. 1993, 65, 645, https://doi.org/10.1021/ ac00053a027.

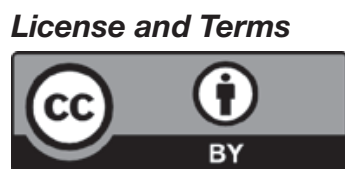

This is an Open Access article under the terms of the Creative Commons Attribution License CC BY 4.0. The material may not be used for commercial purposes.

The license is subject to the CHIMIA terms and conditions: ((https://chimia.ch/chimia/about).

The definitive version of this article is the electronic one that can be found at https://doi.org/10.2533/chimia.2022.114 